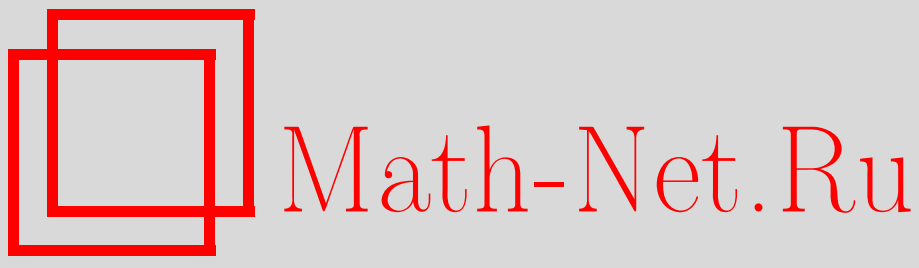

М. Д. Ковалёв, О восстановимости шарнирников по внутренним напряжениям, Изв. РАН. Сер. матем., 1997, том 61, выпуск 4, 37-66

DOI: https://doi.org/10.4213/im135

Использование Общероссийского математического портала Math-Net.Ru подразумевает, что вы прочитали и согласны с пользовательским соглашением

http://www . mathnet.ru/rus/agreement

Параметры загрузки:

IP: 3.85 .7 .115

26 апреля 2023 г., $16: 29: 43$ 
УДК $514+531.8$

\author{
М. Д. Ковалёв
}

\title{
О восстановимости шарнирников по внутренним напряжениям
}

\begin{abstract}
Рассматривается совокупность $\mathfrak{P}$ конструкций (шарнирников) в $\mathbb{R}^{d}$ из рычагов, связанных универсальными шарнирами, с заданной схемой соединения и заданными точками закрепления некоторых шарниров. Исследуется вопрос: в каких случаях шарнирник $\mathbf{p} \in \mathfrak{P}$ можно восстановить по его пространству $W(\mathbf{p})$ внутренних напряжений? Иными словами, когда в совокупности $\mathfrak{P}$ не существует других шарнирников с тем же пространством $W(\mathbf{p})$ напряжений, что и у $\mathbf{p}$ ? Полный ответ получен лишь для шарнирников на прямой. Изучены также геометрические свойства образа рычажного отображения, связанные с шарнирниками, допускающими внутренние напряжения.

Библиография: 7 наименований.
\end{abstract}

\section{§1. Постановка задачи}

Рассмотрим в плоскости простейшую шарнирную конструкцию (шарнирник), состоящую из двух стержней (рычагов), скрепленных между собой шарниром $\mathbf{p}_{1}$ (рис. 1), а другими концами закрепленных с помощью шарниров $\mathbf{p}_{2}$ и $\mathbf{p}_{3}$. Ненулевой набор $\left\{\omega_{12}, \omega_{13}\right\}$ вешественных чисел, сопоставленных рычагам $\mathbf{p}_{1} \mathbf{p}_{2}$ и $\mathbf{p}_{1} \mathbf{p}_{3}$ нашего шарнирника, называют набором его внутренних напряжений, или просто напряжений, в том случае, когда вьполнено следующее условие:

$$
\omega_{12}\left(\mathbf{p}_{2}-\mathbf{p}_{1}\right)+\omega_{13}\left(\mathbf{p}_{3}-\mathbf{p}_{1}\right)=0
$$

где $\mathbf{p}_{1}, \mathbf{p}_{2}, \mathbf{p}_{3}$ - радиус-векторы свободного и закрепленных в плоскости шарниров. Физически это условие выражает равновесие сил, приложенных к свободному шарниру со стороны рычагов $\mathbf{p}_{1} \mathbf{p}_{2}$ и $\mathbf{p}_{1} \mathbf{p}_{3}$ нашего шарнирника. Величины напряжений $\omega_{i j}$ указывают меру напряженности рычагов: если $\omega_{i j}>0$, то рычаг $\mathbf{p}_{i} \mathbf{p}_{j}$ растянут, если же $\omega_{i j}<0$, то он сжат.

Очевидно, наш шарнирник допускает внутренние напряжения лишь в том случае, когда свободный шарнир $\mathbf{p}_{1}$ лежит на прямой $\mathbf{p}_{2} \mathbf{p}_{3}$, соединяющей закрепленные шарниры. Если ось абсцисс декартовой системы координат выбрать на прямой $\mathbf{p}_{2} \mathbf{p}_{3}$, а начало системы в шарнире $\mathbf{p}_{2}$, то условие (1) примет вид

$$
\omega_{12} x_{1}+\omega_{13}\left(x_{1}-x_{3}\right)=0
$$




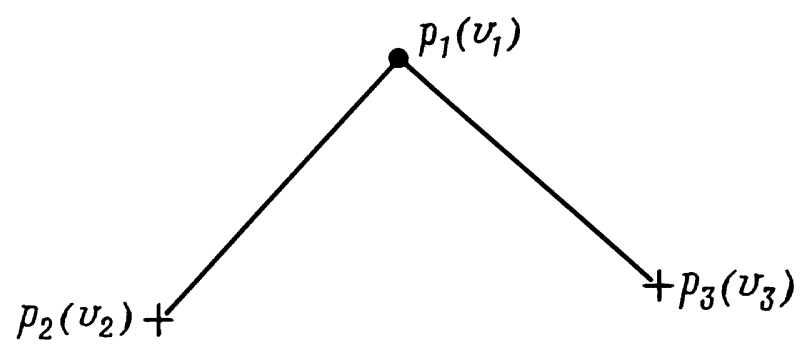

Рис. 1

Будем считать $\mathbf{p}_{2}$ и $\mathbf{p}_{3}$ геометрически различными, т.е. $x_{3} \neq 0$. Тогда допущение $\omega_{12}+\omega_{13}=0$ приводит к $\omega_{13}=0$, а следовательно, и $\omega_{12}=0$. Таким образом, случай внутренних напряжений вида $\omega_{12}=-\omega_{13}$ не осуществляется ни для какого шарнирника с заданной схемой и несовпадающими закрепленными шарнирами. Задание допустимого $\left(\omega_{12} \neq-\omega_{13}\right)$ набора напряжений и несовпадающих между собой закрепленных шарниров однозначно определяет положение $x_{1}=\frac{\omega_{13} x_{3}}{\omega_{12}+\omega_{13}}$, $y_{1}=0$ свободного шарнира, а значит, и весь шарнирник.

В этой статье нас будет интересовать связь структуры шарнирника с возможностью однозначного его восстановления по допустимым наборам внутренних напряжений, если закрепленные шарниры заданы. Прежде чем точно поставить и исследовать этот вопрос в общем виде, введем необходимые определения (см. также [1]).

Шарнирной структурной схемой мы называем связный граф̆ $G(V, E)$ без петель и кратных ребер, множество $V$ вершин которого состоит из двух непустых подмножеств:

$$
V_{1}=\left\{v_{1}, \ldots, v_{m}\right\}, \quad V_{2}=\left\{v_{m+1}, \ldots, v_{m+n}\right\}
$$

удовлетворяюший тем условиям, что его подграф $G_{1}\left(V_{1}, E_{1}\right)$, порожденный множеством $V_{1}$, связен, а вершины множества $V_{2}$ несмежны между собой. Таким образом, множество $E$ ребер графа $G(V, E)$ распадается на два подмножества: $E_{1}$, состоящее из ребер $v_{i} v_{j}$, для которых $i, j \leqslant m$, и $E_{2}$, состояшее из ребер $v_{i} v_{j}, i \leqslant m$, $j>m$.

Далее вершины из множества $V_{1}$ мы будем называть свободными иарнирами, из множества $V_{2}$ - закрепленными шарнирами, а ребра графа $G$ - рычагами, что определяется механическим смыслом нашей модели. Закрепленной в евклидовом пространстве $\mathbb{R}^{d}$ шарнирной схемой (ЗШС в $\mathbb{R}^{d}$ ) назовем структурную схему, каждому закрепленному шарниру $v_{i}$ которой сопоставлена некоторая точка $\mathbf{p}_{i} \in \mathbb{R}^{d}$. Если теперь сопоставить и каждому свободному шарниру свою точку евклидова пространства, то мы получим конкретный шарнирник $\mathbf{p}$, отвечаюший заданной ЗШС в $\mathbb{R}^{d}$. При этом не исключается геометрическое совпадение любых двух шарниров. 
Условие равновесия сил, приложенных к $i$-му свободному шарниру шарнирника со стороны остальных его шарниров, имеет вид

$$
\sum_{j} \omega_{i j}\left(\mathbf{p}_{j}-\mathbf{p}_{i}\right)=\mathbf{0}
$$

где суммирование производится по всем шарнирам, смежным с $\mathbf{p}_{i}$, т.е. соединенным с ним рычагом, $\omega_{i j}=\omega_{j i}$-внутреннее напряжение рычага $\mathbf{p}_{i} \mathbf{p}_{j}$. Если в $\mathbb{R}^{d}$ задан шарнирник $\mathbf{p}=\left(\mathbf{p}_{1}, \ldots, \mathbf{p}_{m+n}\right)$, то его внутренние напряжения $\omega_{i j}$ определяются как нетривиальные решения однородной системы линейных уравнений вида (2):

$$
\sum_{j} \omega_{i j}\left(\mathbf{p}_{j}-\mathbf{p}_{i}\right)=\mathbf{0}, \quad 1 \leqslant i \leqslant m
$$

Таким образом, множество внутренних напряжений заданного шарнирника вместе с нулевым напряжением представляет собой линейное подпространство $W(\mathbf{p})$ евклидова пространства $W^{r}, r=|E|$, всех мыслимых напряжений. Когда это подпространство нульмерно, множество внутренних напряжений пусто и мы говорим, что данный шарнирник не допускает напряжений. В рассмотренном примере таковыми являются шарнирники с $\mathbf{p}_{1}=\left(x_{1}, y_{1}\right)$, где $y_{1} \neq 0$. Заметим, что шарнирники, не допускающие внутренних напряжений, возможны лишш в случае $r \leqslant d m$.

Для заданной ЗШС, как мы видели, могут сушествовать наборы напряжений, не реализуюшиеся ни при каком выборе положений свободных шарниров. Такие наборы мы называем недопустимылми для данной ЗШС в отличие от остальных, допустимых наборов.

Справедливо следующее

УТВЕРЖДЕНИЕ 1. Любое внутреннее напряжение допускают те и только те ЗШС, все закрепленные шарниры которых совпадают между собой.

Действительно, если все закрепленные шарниры лежат в одной точке, то, поместив и все свободные шарниры в эту же точку, мы получим систему уравнений (3) относительно $\omega_{i j}$ с нулевой матрищей. Решением этой системы является произвольный набор внутренних напряжений. Пусть теперь ЗШС содержит по крайней мере два шарнира, закрепленных в разных точках $\mathbf{p}_{a}$ и $\mathbf{p}_{b}$. В силу связности имеется простая цепь из рычагов ЗШС, соединяющая между собой эти закрепленные шарниры и проходяшая лишь через свободные шарниры. Пусть, для определенности, это цепь $v_{a}, v_{1}, \ldots, v_{k}, v_{b}$. Составим набор внутренних напряжений из напряжений $\omega_{a 1}=1, \omega_{12}=-0.5, \omega_{23}=0.5, \ldots, \omega_{k-1 k}=(-1)^{k-1} \cdot 0.5, \omega_{k b}=(-1)^{k}$ чередуюшегося знака на рычагах этой цепи и равных нулю на всех остальных рычагах. Подставим эти напряжения в систему (3). Нетривиальные уравнения будут отвечать лишь шарнирам $v_{1}, \ldots, v_{k}$. Расположив их в этом же порядке, умножив $i$-ое из них на $(-1)^{i-1}$ и сложив, получим

$$
\left(\mathbf{p}_{1}-\mathbf{p}_{a}\right)+\left(\mathbf{p}_{2}-\mathbf{p}_{1}\right)+\cdots+\left(\mathbf{p}_{b}-\mathbf{p}_{k}\right)=\mathbf{p}_{b}-\mathbf{p}_{a}=\mathbf{0}
$$


Последнее противоречит предположению.

Если задана ЗШС в $\mathbb{R}^{d}$, а также набор внутренних напряжений, то систему уравнений (3) можно переписать так:

$$
\left(\sum_{\substack{j \\ v_{i} v_{j} \in E}} \omega_{i j}\right) \mathbf{p}_{i}-\sum_{\substack{j \\ v_{i} v_{j} \in E_{1}}} \omega_{i j} \mathbf{p}_{j}=\sum_{\substack{j \\ v_{i} v_{j} \in E_{2}}} \omega_{i j} \mathbf{p}_{j}, \quad 1 \leqslant i \leqslant m .
$$

Здесь неизвестные - радиус-векторы свободных шарниров - содержатся в левой части уравнений. Матрица $\Omega$ этой системы является симметрической; ее элемент $i$-ой строки, стояший на главной диагонали, равен $\sum_{v_{i} v_{j} \in E}^{j} \omega_{i j}$, прочие же элементы равны $-\omega_{i j}$, если шарниры $v_{i}$ и $v_{j}$ смежны в ЗШС, и нулю в противном случае. Заметим еще, что векторная система уравнений (4) в координатах расписывается как $\Omega^{*} X=B$, где $X$ - столбец, составленньй из координат $\left(x_{i}^{1}, \ldots, x_{i}^{d}\right)$ точек $\mathbf{p}_{i}$ следуюшим образом:

$$
\left(x_{1}^{1}, x_{2}^{1}, \ldots, x_{m}^{1}, x_{1}^{2}, \ldots, x_{m}^{2}, \ldots, x_{1}^{d}, \ldots, x_{m}^{d}\right)
$$

$\Omega^{*}$ - симметрическая $(d m \times d m)$-матрища, представляющая собой прямое произведение матрищы $\Omega$ на $d$-мерную единичную матрищу, $B$ - столбец правых частей, взятых в том же порядке, что и элементы столбца $X$.

Далее мы будем рассматривать совокупности шарнирников с фиксированной ЗШС и параметризовать их точками $\mathbf{p}=\left(\mathbf{p}_{1}, \mathbf{p}_{2}, \ldots, \mathbf{p}_{m}\right) \in \mathbb{R}^{d m}$. Пусть $W\left(\mathbf{p}^{0}\right)-$ пространство внутренних напряжений шарнирника $\mathbf{p}^{0}=\left(\mathbf{p}_{1}^{0}, \mathbf{p}_{2}^{0}, \ldots, \mathbf{p}_{m}^{0}\right)$, т.е. $W\left(\mathbf{p}^{0}\right)$ есть множество решений $\left\{\omega_{i j}\right\}$ системы уравнений $(3)$ при заданных $\mathbf{p}_{1}=$ $\mathbf{p}_{1}^{0}, \ldots, \mathbf{p}_{m}=\mathbf{p}_{m}^{0}$, и, разумеется, $\mathbf{p}_{m+1}=\mathbf{p}_{m+1}^{0}, \ldots, \mathbf{p}_{m+n}=\mathbf{p}_{m+n}^{0}$. Наш основной вопрос: в каких случаях исходный шарнирник $\mathbf{p}^{0}$ восстанавливается однозначно по подпространству $W\left(\mathbf{p}^{0}\right) \in W^{r}$ при заданной ЗШС? (Здесь важно расположение подпространства относительно стандартной системы координат $\left(\omega_{i j}\right)$ в $W^{r}$.) Иными словами, если $\mathbf{p}(\omega)=\left(\mathbf{p}_{1}(\omega), \ldots, \mathbf{p}_{m}(\omega)\right)$ есть множество всех решений системы линейных уравнений (4) при некотором $\left\{\omega_{i j}\right\}=\omega \in W\left(\mathbf{p}^{0}\right)$, то когда

$$
\bigcap_{\omega \in W\left(\mathbf{p}^{0}\right)} \mathbf{p}(\omega)=\mathbf{p}^{0} ?
$$

Очевидно, условие $\operatorname{det} \Omega(\omega) \neq 0$, где $\omega$ - какой-либо ненулевой вектор из $W\left(\mathbf{p}^{0}\right)$, является достаточным для восстановимости шарнирника $\mathbf{p}^{0}$ по пространству его напряжений. В случае одномерного пространства $W\left(\mathbf{p}^{0}\right)$ это условие является и необходимым. Однако нас будут интересовать, главным образом, условия геометрического характера на шарнирник $\mathbf{p}^{0}$ и его ЗШС, а также на подпространство $W\left(\mathbf{p}^{0}\right) \subset W^{r}$, позволяюшие ответить на вопрос о восстановимости этого шарнирника без вычисления $\operatorname{det} \Omega(\omega)$. Примером такого достаточно общего признака 
является следующий: если найдется напряжение $\omega^{0} \in W\left(\mathbf{p}^{0}\right)$, положительное на каждом рычаге шарнирника, то шарнирник $\mathbf{p}^{0}$ восстановим по своему пространству напряжений. Такие шарнирники исследовались в работе [2], мы будем называть их паутинным.м. В этом случае независимо от структуры $3 Ш \mathrm{C} \operatorname{det} \Omega\left(\omega^{0}\right) \neq 0$, и шарнирник восстанавливается по напряжению $\omega^{0}$.

Назовем шарнирник сократимым, если хотя бы два его смежных шарнира совпадают, и несократимым в противном случае. Заметим, что если шарнирник $\mathbf{p}^{0}$ сократимый, т.е. $\mathbf{p}_{i}^{0}=\mathbf{p}_{j}^{0}$, то его пространство внутренних напряжений содержит вектор $\omega^{0}$ с одной лишь ненулевой координатой $\omega_{i j}^{0}$. Верно и обратное. В нашей модели усилие через рычаг $\mathbf{p}_{i}^{0} \mathbf{p}_{j}^{0}$ нулевой длины передаваться не может, что противоречит “физическому смыслу". В таких случаях можно рассмотреть шарнирник с более простой ЗШС, полученной из исходной отождествлением вершин, отвечаюших совмешенным шарнирам. Нам тоже удобно будет исключить этот случай и рассматривать в дальнейшем несократимые шарнирники.

Если для шарнирника $\mathbf{p} \omega_{i j}=0$ при любом напряжении $\omega \in W(\mathbf{p})$, то рычаг $\mathbf{p}_{i} \mathbf{p}_{j}$ этого шарнирника мы назовем ненапрягаемым. Легко уяснить, что при удалении из шарнирника $\mathbf{p}$ одного рычага может получиться либо один $\left(\mathbf{p}^{\prime}\right)$, либо два $\left(\mathbf{p}^{\prime}\right.$ и $\left.\mathbf{p}^{\prime \prime}\right)$ новых шарнирника.

УТВЕРЖДЕНИЕ 2. Шарнирник $\mathbf{p}$, имеющий ненапрягаемый рычаг $\mathbf{p}_{i} \mathbf{p}_{j}$, восстановим (по своему пространству внутренних напрязений) тогда и только тогда, когда восстановим шарнирник $\mathbf{p}^{\prime}$ либо каждый из шарнирников $\mathbf{p}^{\prime}$ u $\mathbf{p}^{\prime \prime}$ в случае распадения шарнирника $\mathbf{p}$ при удалении рычага $\mathbf{p}_{i} \mathbf{p}_{j}$.

Рассмотрим первый случай. В этом случае пространства $W(\mathbf{p})$ и $W\left(\mathbf{p}^{\prime}\right)$ напряжений шарнирников $\mathbf{p}$ и $\mathbf{p}^{\prime}$ можно отождествить как подпространства пространства $W^{r}(r$ - число рычагов шарнирника). Поэтому совпадают совокупности систем (4), отвечающих всем $\omega \in W(\mathbf{p})$ и всем $\omega^{\prime} \in W\left(\mathbf{p}^{\prime}\right)$, а значит, и пересечения (5) всех их решений.

Таким образом, перейдя, если нужно, к более простой ЗШС, можно ограничить рассмотрение задачи восстановления несократимыми и полностью напрягаемыми шарнирниками. Полностью напрягаемым мы называем шарнирник, не имеющий ненапрягаемых рычагов. Это условие, как легко видеть, равносильно сушествованию внутреннего напряжения, ненулевого на каждом рычаге шарнирника. Заметим еще, что рычаг $\mathbf{p}_{i} \mathbf{p}_{j}$ не напрягаем ни в одном несократимом шарнирнике $\mathbf{p} c$ данной ЗШС тогда и только тогда, когда ребро $v_{i} v_{j}$ не содержится ни в одном из циклов граффа этой ЗШС, а также ни в одной из цепей с концами в закрепленных шарнирах.

Однако условий несократимости и полной напрягаемости отнюдь не достаточно для восстановимости шарнирника. Если все закрепленные шарниры шарнирника $\mathbf{p}$ лежат в некотором линейном подмногообразии $L \subset \mathbb{R}^{d}$, а свободные шарниры не все лежат в $L$, то имеется афффинное преобразование $\mathbb{A}$ пространства $\mathbb{R}^{d}$, переводящее шарнирник $\mathbf{p}$ в отличный от него шарнирник $\mathbb{A} \mathbf{p}$ с той же самой ЗШС. Это преобразование можно выбрать из аффинных преобразований $\mathbb{R}^{d}$, оставляющих 
все точки многообразия $L$ на месте. Из вида системы (4) следует, что пространство внутренних напряжений шарнирника $\mathbb{A} \mathbf{p}$ совпадает с пространством напряжений шарнирника р. Более общее условие невосстановимости шарнирника устанавливается теоремой 2 из $\S 3$.

В следующем параграфе будет доказано необходимое и достаточное условие восстановимости несократимого полностью напрягаемого шарнирника на прямой. Это - несложное условие на его ЗШС. По существу, все случаи невосстановимости несократимого полностью напрягаемого шарнирника на прямой исчерпьваются описанными выше и обусловливаются особенностями его ЗШС. Положение в корне усложняется уже на плоскости. В $\S 3$ приведен пример $3 Ш С$ в $\mathbb{R}^{2}$, для которой существуют два несократимых полностью напрягаемых шарнирника $\mathbf{p}$ и $\mathbf{p}^{\prime}$ с одномерными пространствами внутренних напряжений, один из которых восстановим по своему пространству напряжений, а другой - нет. Более того, невосстановимьй шарнирник не удовлетворяет условиям теоремы 2.

В $\S 4$ исследуется связь восстановимости шарнирников по внутренним напряжениям с геометрическими свойствами образа рычажного отображения (см. [1]), представляющими и самостоятельный интерес. А также рассматриваются с этой точки зрения паутинные шарнирники и доказывается сушествование восстановимых, возможно, сократимых, шарнирников для произвольной ЗШС. В $\S 5$ установлены условия, влекущие существование несократимых восстановимых шарнирников с данной ЗШС. В заключении приведены нерешенные вопросы.

\section{§2. Шарнирники на прямой}

Для шарнирников в $\mathbb{R}^{1}$ система (3) является обычной скалярной системой линейных уравнений:

$$
\sum_{j} \omega_{i j}\left(x_{j}-x_{i}\right)=0, \quad 1 \leqslant i \leqslant m
$$

где $x_{i}$ - координата $i$-го шарнира на прямой.

ЛЕмма 1. Пространство внутренних напряжений несократимого шарнирника на прямой с $m$ свободнымми шарнирами и $r$ рычагами имеет размерность $r-m$.

ДоказАтельство. Достаточно показать, что ранг матрицы $M$ системы уравнений $(6)$ относительно неизвестных $\omega_{i j}$ равен числу $m$ ее строк. Пусть $G$ - граф нашей ЗШС, а $G_{1}$ - ее подграф, порожденный свободными шарнирами. В силу связности графа $G_{1}$ из него можно выделить остовное дерево $D_{1}$. Добавив к дереву $D_{1}$ один из закрепленных шарниров графа $G$ вместе с исходяшим из него рычагом, получим дерево $D$, содержащее $m+1$ вершин и $m$ ребер. Возьмем за корневую вершину дерева $D$ закрепленньй шарнир и перенумеруем его вершины каким-либо способом, не противоречашим естественному частичному порядку вершин в дереве (рис. 2). Рассмотрим минор $M^{*}$ порядка $m$ матрищы $M$, содержаший столбцы, 
отвечающие ребрам дерева $D$. Если его столбцы расположить в лексикографическом порядке, то ниже главной диагонали окажутся лишь нулевые элементы, а на главной диагонали - разности $x_{j}-x_{i}$. В силу несократимости шарнирника $\mathbf{p}^{0}$ минор $M^{*}=\left(x_{1}-x_{0}\right)\left(x_{2}-x_{1}\right) \ldots\left(x_{m}-x_{k}\right)$ не равен 0 . Лемма доказана.

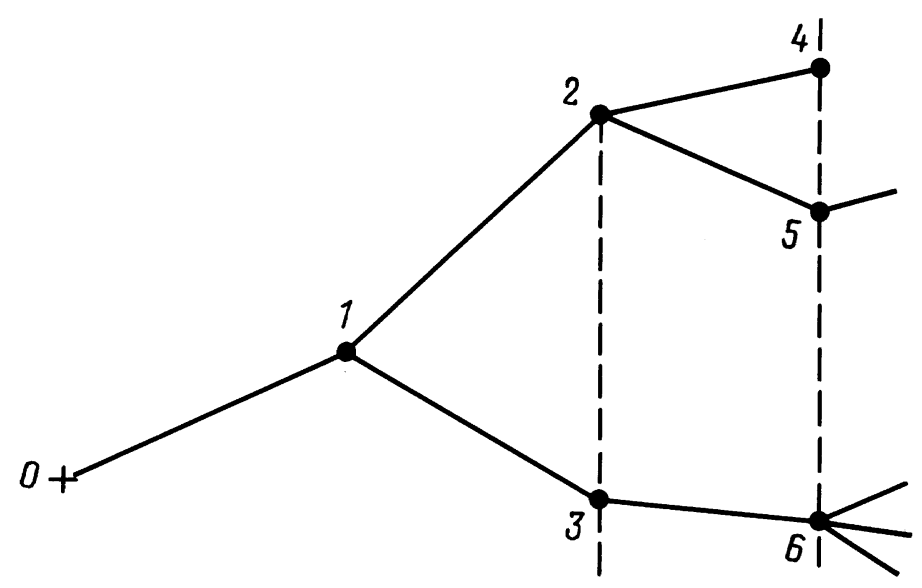

Рис. 2

Перейдем к формулировке основной теоремы. Допустим, что у исходной ЗШС в $\mathbb{R}^{1}$ есть закрепленные шарниры, совпадаюшие между собой как точки прямой. Отождествляя друг с другом эти совпавшие шарниры, мы получим новую ЗШС $\mathfrak{Z}$ с меньшим числом закрепленных шарниров. Будем называть ее приведенной ЗШС. Пусть $G=G(V, E),|E|=r$, - ее граф. Если приведенная ЗШС имеет более одного закрепленного шарнира: $v_{m+1}, \ldots, v_{m+n}$, то, добавив к ее графу ребра $v_{m+1} v_{m+2}, \ldots, v_{m+1} v_{m+n}$, соединяюшие первый из закрепленных шарниров с остальными, мы получим граф $G^{\prime}\left(V, E^{\prime}\right)$. Назовем его дополненным графом исходной ЗШС. Вершину $v \in G^{\prime}$ назовем разделяющеей, если при удалении ее вместе с инцидентными ей ребрами из графа $G^{\prime}$ получается несвязный граф̆. Наличие хотя бы одной разделяющей вершины в графе $G^{\prime}$ равносильно тому, что число вершинной связности $\varkappa\left(G^{\prime}\right)$ равно 1.

Tеорема 1. Для восстановимости несократимого шарнирника $\mathbf{p}^{0}$ на прямой по его подпространству $W\left(\mathbf{p}^{0}\right) \in W^{r}$ внутренних напряжений необходимо и достаточно выполнения следующих двух условий:

1) граф $G$ приведенной ЗШС Z шарнирника имеет более одного закрепленного иарнира;

2) дополненный граф $G^{\prime}$ ЗШС не содержит разделяющих вершин.

Для доказательства теоремы нам понадобится циклическое расщепление системы уравнений (6), которое мы сейчас опишем. Наибольшее число независимых 
циклов в графе $G^{\prime}\left(V, E^{\prime}\right)$ (цикломатическое число графа) равно

$$
\left|E^{\prime}\right|-|V|+1=r+n-1-(m+n)+1=r-m=\nu .
$$

Выберем в графе $G^{\prime}$ остовное дерево $D$, содержащее ребра $v_{m+1} v_{m+2}$, $v_{m+1} v_{m+3}, \ldots, v_{m+1} v_{m+n}$. С этим деревом ассоциирована [3] фундаментальная система циклов $\mathbf{C}=\left\{C_{1}, C_{2}, \ldots, C_{\nu}\right\}$, причем имеется естественное взаимно однозначное соответствие между ребрами из $G^{\prime} \backslash D$ и циклами из $\mathbf{C}$, а именно, каждое из этих ребер принадлежит лишь одному из циклов совокупности $\mathbf{C}$.

Лемма 2. Множсество $\mathbf{C}$ можно упорядочить так, что первые $n-1$ чиклов будут содержать по крайней мере по два закрепленных шарнира, а остальные (если они существуют) пересекаться с облединением всех предшествующих по крайней мере по ребру.

ДокАЗАТЕЛЬСТво. Будем считать вершину $(m+1)$ корневой в дереве $D$. Из этой вершины исходит по крайней мере $n$ ветвей $\mathscr{V}_{1}, \ldots, \mathscr{V}_{n}: n-1$ ветвь - в остальные закрепленные шарниры и по крайней мере одна в свободный шарнир. Ветвью мы называем компоненту связности графа, полученного удалением из $D$ корневой вершины вместе с инцидентньми ей ребрами. Поскольку граф $G$ связен, то сушествует не менее $n-1$ ребер из $G \backslash D$, связывающих между собой ветви $\mathscr{V}_{1}, \ldots, \mathscr{V}_{n}$. Каждому такому ребру отвечает цикл, содержащий либо два, либо три закрепленных шарнира. Таким образом, возможность выбора циклов $C_{1}, \ldots, C_{n-1}$ доказана.

Пусть $\mathscr{G}=\bigcup_{i=1}^{n-1} C_{i}$, рассмотрим множество $\mathscr{L}$ ребер дерева $D$, не принадлежаших $\mathscr{G}$. Очевидно, если $\mathscr{L}$ не пусто, то оно является лесом. Пусть дерево $\mathscr{D}$ этого леса имеет корневую вершину $v \in \mathscr{G}$. Поскольку в графе $G^{\prime}$ нет разделяюших вершин, то непременно найдется ребро $e \in G^{\prime}$, соединяющее какую-либо отличную от корневой вершину дерева $\mathscr{D}$ либо с вершиной $v^{\prime} \in \mathscr{G}, v^{\prime} \neq v$, либо с вершиной $v^{\prime}$, принадлежащей другому дереву леса $\mathscr{L}$. В обоих случаях цикл $C_{n}$, отвечающий ребру $е$, содержит по крайней мере одно ребро графа $\mathscr{G}$.

Это рассуждение можно повторять до тех пор, пока не получим пустого леса $\mathscr{L}$. Если объединение $\mathscr{G}^{\prime}$ всех полученных при этом циклов не содержит всех ребер графа $G^{\prime}$, то оставшимся ребрам графа $G^{\prime}$, очевидно, отвечают циклы, пересекаюшиеся с $G^{\prime}$, а значит, и с объединением предыдущих циклов, не менее чем по двум ребрам. Лемма доказана.

Совокупности $\mathbf{C}$ циклов можно сопоставить совокупность $\mathbf{S}=\left\{S_{1}, S_{2}, \ldots, S_{\nu}\right\}$ систем линейных уравнений, каждая из которых отвечает равновесию сил в свободных шарнирах соответствуюшего цикла. Например, для цикла $C$ : $x_{1}, x_{2}, \ldots, x_{p}, x_{1}$, все шарниры которого свободны, соответствуюшая система $S$ 
выглядит так:

$$
\begin{aligned}
& \omega_{1 p}\left(x_{1}-x_{p}\right)+\omega_{12}\left(x_{1}-x_{2}\right)=0, \\
& \omega_{21}\left(x_{2}-x_{1}\right)+\omega_{23}\left(x_{2}-x_{3}\right)=0, \\
& \ldots \ldots \ldots \ldots \ldots \ldots \ldots \ldots \ldots \ldots \ldots \\
& \omega_{p p-1}\left(x_{p}-x_{p-1}\right)+\omega_{p 1}\left(x_{p}-x_{1}\right)=0 .
\end{aligned}
$$

Если же в цикле $C$ имеются закрепленные шарниры, то в нашем случае они входят в цикл $C$ в одном месте подряд, и уравнения, отвечающие им, надо исключить из системы (7). После такого исключения получается система уравнений несколько иного вида: например, в случае закрепленных шарниров $x_{1} x_{p}$ система вида

$$
\begin{aligned}
& \omega_{21}\left(x_{2}-x_{1}\right)+\omega_{23}\left(x_{2}-x_{3}\right)=0, \\
& \omega_{32}\left(x_{3}-x_{2}\right)+\omega_{34}\left(x_{3}-x_{4}\right)=0, \\
& \omega_{p-1 p-2}\left(x_{p-1}-x_{p-2}\right)+\omega_{p-1 p}\left(x_{p-1}-x_{p}\right)=0 .
\end{aligned}
$$

Далее решение системы $S_{\sigma}, 1 \leqslant \sigma \leqslant \nu$, мы будем записывать в виде вектора $\omega_{\sigma} \in W^{r}$, полагая его координаты $\omega_{i j}$, не вошедшие в систему $S_{\sigma}$, равными нулю.

ЛЕмма 3. Для несократимого шарнирника $\mathbf{p}^{0}$ решение каждой из систем $S_{\sigma}$ имеет вид $\alpha_{\sigma} \omega_{\sigma}, \alpha_{\sigma} \in \mathbb{R}$, причем векторы $\omega_{\sigma}, 1 \leqslant \sigma \leqslant \nu$, линейно независимы и их линейная оболочка $W$ совпадает с пространством $W\left(\mathbf{p}^{0}\right)$ внутренних напряжений шарнирника.

ДокаЗАтельство. Решение $\omega$ системы уравнений (7) имеет вид

$$
\omega_{1 p}, \quad \omega_{i i+1}=\frac{x_{1}-x_{p}}{x_{i+1}-x_{i}} \omega_{1 p}, \quad 1 \leqslant i \leqslant p-1
$$

т.е. является однопараметрическим. То же самое можно сказать и о решениях систем вида $\left(7^{\prime}\right)$. Таким образом, каждая из систем $S_{\sigma}$ имеет однопараметрическое семейство решений $\alpha_{\sigma} \omega_{\sigma}$. Причем у вектора $\omega_{\sigma}$ все координаты $\omega_{i j}$, отвечающие рычагам цикла $C_{\sigma}$, содержашимся в графе $G$, ненулевые, все же прочие координаты равны нулю.

Очевидным образом каждый из векторов $\omega_{\sigma}$, а значит, и произвольная их линейная комбинация удовлетворяют системе уравнений (6). Итак, $W \subseteq W\left(\mathbf{p}^{0}\right)$. Поскольку вследствие леммы $1 \operatorname{dim} W\left(\mathbf{p}^{0}\right)=r-m=\nu$, то достаточно удостовериться в линейной независимости векторов $\omega_{\sigma}$.

Допустим противное, т.е. $\sum_{\sigma=1} \alpha_{\sigma} \omega_{\sigma}=0$, где для определенности $\alpha_{1} \neq 0$. Так как в цикле $C_{1}$ имеется ребро $(\gamma, \beta)$, не входящее ни в один из прочих циклов, то координата выписанной линейной комбинации, отвечающая этому ребру, равна $\alpha_{1} \omega_{\gamma \beta} \neq 0$. Последнее противоречит нашему допущению. 
ДокаЗАТЕЛЬСТво ТеОремы 1. ДостАточность. Пусть $W\left(\mathbf{p}^{0}\right)-$ пространство напряжений несократимого шарнирника $\mathbf{p}^{0}$ на прямой с приведенной ЗШС $\mathfrak{Z}$, удовлетворяющей условиям теоремы. Обозначим $\mathbf{p}(\omega)=\left\{x_{1}(\omega), \ldots, x_{m}(\omega)\right\}$ множество решений системы $\mathscr{S}(\omega)$ уравнений $(6)$ при заданном $\omega \in W\left(\mathbf{p}^{0}\right)$, а $\mathbf{p}^{1}=\bigcap_{\omega \in W\left(\mathbf{p}^{0}\right)} \mathbf{p}(\omega)$. Очевидно, $\mathbf{p}^{1}$ является линейным многообразием, а равенство $\mathbf{p}^{1}=\mathbf{p}^{0}$ равносильно восстановимости шарнирника $\mathbf{p}^{0}$ по его пространству напряжений. Для доказательства равенства $\mathbf{p}^{1}=\mathbf{p}^{0}$ мы воспользуемся циклическим расщеплением системы уравнений (6). Как только что было установлено, несократимый шарнирник $\mathbf{p}^{0}$ вместе с фундаментальной системой $\mathbf{C}$ циклов графа $G^{\prime}$ определяет базис из векторов $\omega_{\sigma}, 1 \leqslant \sigma \leqslant \nu$, в пространстве $W\left(\mathbf{p}^{0}\right)$. Хотя, не зная шарнирника $\mathbf{p}^{0}$, мы не можем указать этого базиса, мы будем пользоваться фактом его существования. Каждому из векторов $\omega_{\sigma}$ этого базиса, определенному с точностью до умножения на ненулевое число, отвечает своя система линейных уравнений $S_{\sigma}$ вида $(7)$ или $\left(7^{\prime}\right)$, также определенная с точностью до умножения всех уравнений на тот же ненулевой множитель. На этот раз неизвестными в системах $S_{\sigma}$ мы считаем $x_{i}$. Заметим, что множество $\mathbf{p}_{\sigma} \subset \mathbb{R}^{m}$ решений системы уравнений $S_{\sigma}$ состоит из векторов $\mathbf{p}=\left(x_{1}, \ldots, x_{m}\right)$, все координаты которых, не вошедшие в систему $S_{\sigma}$, произвольны. Пусть решением совокупности $\mathbf{S}$ систем $S_{\sigma}$ является линейное многообразие $\mathbf{p}^{2}=\bigcap_{\sigma=1}^{\nu} \mathbf{p}_{\sigma}$.

Лемма 4. Многообразие $\mathbf{p}^{1}$ совпадает смногообразием $\mathbf{p}^{2}$.

ДокАЗАТЕльСтво. Допустим, что вектор $\mathbf{p} \in \mathbb{R}^{m}$ удовлетворяет каждой из систем $\mathscr{S}(\omega), \omega \in W\left(\mathbf{p}^{0}\right)$. Поскольку $\omega=\sum_{\sigma=1}^{\nu} \alpha_{\sigma} \omega_{\sigma}$, где $\alpha_{\sigma}$ могут быть произвольными, то р удовлетворяет каждой из систем $\mathscr{S}\left(\omega_{\sigma}\right)$, совпадающей, очевидно, с соответствующей системой $S_{\sigma}$. Таким образом, $\mathbf{p}^{1} \subset \mathbf{p}^{2}$.

Пусть теперь вектор р удовлетворяет каждой из систем $S_{\sigma}, 1 \leqslant \sigma \leqslant \nu$, а $\omega=\sum_{\sigma=1}^{\nu} \alpha_{\sigma} \omega_{\sigma}-$ произвольная линейная комбинация векторов $\omega_{\sigma}$. Поскольку каждое уравнение системы $\mathscr{S}(\omega)$ представляет собой линейную комбинацию уравнений систем $S_{\sigma}$, умноженных на $\alpha_{\sigma}$, то вектор р удовлетворяет и системе $\mathscr{S}(\omega)$ при произвольном $\omega \in W\left(\mathbf{p}^{0}\right)$. Итак, $\mathbf{p}^{1} \supset \mathbf{p}^{2}$, а следовательно, и $\mathbf{p}^{1}=\mathbf{p}^{2}$. Лемма доказана.

Остается установить, что в нашем случае многообразие $\mathbf{p}^{2}$ состоит из одной лишь точки $\mathbf{p}^{0}$. Для этого нам понадобится следуюшая лемма.

Рассмотрим системы уравнений $S_{\sigma}(7),\left(7^{\prime}\right)$ относительно неизвестных $x_{i}$ с заданными наборами $\left\{\omega_{i j}\right\}=\omega_{\sigma}$, отвечающими несократимому шарнирнику $\mathbf{p}^{0}$.

ЛЕмма 5. Если в системе вида (7) произвольньм образом задать значения неизвестных $x_{1}$ и $x_{p}$, то она будет иметь единственное решение; если жсе задать значение лишь одного из этих неизвестных, то она будет иметь однопараметрическое семейство решений. Система уравнений вида $\left(7^{\prime}\right)$ всегда имеет единственное решение. 
ДокАЗАТЕЛЬСТВо. По условию при рассматриваемом наборе $\omega_{i j}$ система (7) совместна, и, кроме того, в лемме 3 установлено, что $\omega_{i j} \neq 0$. Решая последовательно уравнения системы (7), получим

$$
\begin{aligned}
& x_{1}-x_{2}=\frac{\omega_{1 p}}{\omega_{12}}\left(x_{p}-x_{1}\right), \quad x_{2}-x_{3}=\frac{\omega_{1 p}}{\omega_{23}}\left(x_{p}-x_{1}\right), \\
& x_{p-2}-x_{p-1}=\frac{\omega_{1 p}}{\omega_{p-2 p-1}}\left(x_{p}-x_{1}\right), \quad x_{p-1}-x_{p}=\frac{\omega_{1 p}}{\omega_{p-1 p}}\left(x_{p}-x_{1}\right) .
\end{aligned}
$$

Если задать значения $x_{1}$ и $x_{p}$, то неизвестные $x_{2}, x_{3}, \ldots, x_{p-1}$ последовательно определяются этими равенствами. Для системы вида $\left(7^{\prime}\right)$ мы имеем ту же самую ситуацию. Если же в системе (7) задать лишь значение $x_{1}$, то неизвестные $x_{2}, x_{3}, \ldots, x_{p}$ можно выразить через одно из них, например через $x_{p}$, и, таким образом, семейство решений в этом случае однопараметрично.

Завершим доказательство теоремы 1 . Мы выбрали фундаментальную систему циклов $\mathbf{C}$ так, что циклам $C_{1}, C_{2}, \ldots, C_{n-1}$ отвечают системы уравнений $S_{1}, \ldots$ $\ldots, S_{n-1}$ вида $\left(7^{\prime}\right)$. Вследствие леммы 5 координаты свободных шарниров этих циклов восстанавливаются по соответствующим наборам $\left\{\omega_{i j}\right\}=\omega_{\sigma}, 1 \leqslant \sigma \leqslant$ $n-1$. Каждый же из циклов $C_{\sigma}, \sigma \geqslant n-1$, пересекаясь, вследствие леммы 2 , с объединением предшествуюших ему циклов не менее чем по ребру, содержит не менее двух шарниров с определенными уже координатами. По лемме 5 ему отвечает система уравнений $S_{\sigma}$, имеюшая единственное решение. Таким образом, по внутренним напряжениям однозначно восстанавливаются положения всех шарниров произвольного цикла $C_{\sigma}$, а поскольку из отсутствия разделяющих вершин в графе $G^{\prime}$ следует, что каждая его вершина принадлежит какому-либо циклу $C_{\sigma}$, то и положения всех свободных шарниров шарнирника $\mathbf{p}^{0}$. Достаточность условия теоремы доказана.

НЕоБХодимость. Пусть нарушено первое условие теоремы, и допустим для определенности, что единственный закрепленный шарнир приведенной ЗШС находится в точке $x_{m+1}=0$. Тогда, как легко видеть, если $\left(x_{1}, \ldots, x_{m}\right)$ есть решение системы уравнений (6) при заданном наборе $\omega_{i j}$, то и $\left(k x_{1}, \ldots, k x_{m}\right)$ при произвольном $k \in \mathbb{R}$ будет решением системы (6) при том же наборе $\omega_{i j}$. Таким образом, условие 1) необходимо для восстановимости шарнирника по его внутренним напряжениям.

Допустим теперь, что не выполнено условие 2$)$ и у графа $G^{\prime}$ есть разделяющая вершина $v_{l}$. Пусть, для определенности, $x_{l}=0$ - координата соответствующего шарнира. Если из графа $G^{\prime}$ удалить вершину $v_{l}$ вместе с инцидентными ей ребрами, то останется граф, состояший не менее чем из двух компонент связности: $G_{1}^{*}, G_{2}^{*}, \ldots$, причем хотя бы одна из этих компонент, пусть $G_{1}^{*}$, не содержит закрепленных шарниров. Тогда если набор $\left(x_{1}, \ldots, x_{m}\right)$ координат свободных шарниров удовлетворяет системе (6) при некотором $\omega$, то и набор $k x_{l}$ при $v_{l} \in G_{1}^{*}$ и $x_{i}$ при $v_{i} \notin G_{1}^{*}$ для произвольного $k \in \mathbb{R}$ удовлетворяет той же самой системе. Теорема доказана. 
Видоизменяя рассуждения, использованные при доказательстве теоремы 1 , можно получить и более общую теорему. Пусть $W\left(\mathbf{p}^{0}\right) \subset W^{r}$ - подпространство внутренних напряжений несократимого полностью напрягаемого шарнирника $\mathbf{p}^{0}$ на прямой с приведенной ЗШС $\mathfrak{Z}, \mathbf{a ~}^{1} \subset \mathbb{R}^{m}$ - многообразие всех шарнирников на прямой с ЗШС $\mathfrak{Z}$ и пространством напряжений $W\left(\mathbf{p}^{0}\right)$. И пусть дополненньй граф $G^{\prime}$ ЗШС $\mathfrak{Z}$ имеет разделяюшие вершины. Рассмотрим максимальные неодносвязные (т.е. с $\varkappa\left(\mathbf{G}_{i}\right)>1$ ) подграфы $\mathbf{G}_{1}, \mathbf{G}_{2}, \ldots, \mathbf{G}_{t}$ графа $G^{\prime}$. Очевидно, каждые два из них пересекаются разве лишь по одной вершине, являющейся разделяющей в графе $G^{\prime}$. Из условия полной напрягаемости шарнирника $\mathbf{p}^{0}$ следует, что каждое ребро и каждая вершина графа $G^{\prime}$ принадлежат некоторому циклу. Поэтому объединение всех этих подграфов совпадает с графом $G^{\prime}$. Мы будем говорить, что граф $G^{\prime}$ распадается на $t$ неодносвязных подграфов.

Теорема $1^{\prime}$. Многообразие $\mathbf{p}^{1}$ q-мерно тогда и только тогда, когда:

а) либо граф $G^{\prime}$ имеет лишь один закрепленный шарнир $\mathbf{p}_{m+1}$ и распадается на q неодносвязных подграфов;

б) либо у графа $G^{\prime}$ имеется не менее двух закрепленных шарниров и он распадается на $q+1$ неодносвязных подграфов.

Приведем набросок доказательства. Вследствие максимальности неодносвязного подграфа $\mathbf{G}_{i}$ в графе $G^{\prime}$ нет циклов, пересекающихся с $\mathbf{G}_{i}$ более, чем по одной вершине и не принадлежащих этому подграфу. Отсюда и из связности графа $G^{\prime}$ следует, что множество подграфов $\mathbf{G}_{i}$ можно упорядочить так, чтобы $\mathbf{G}_{1}$ содержал все закрепленные шарниры ЗШС, а каждый из последующих подграфов $\mathbf{G}_{i}$ пересекался с объединением всех предыдущих ровно по одной вершине.

В случае а) положения свободных шарниров подграфа $\mathbf{G}_{1}$ однозначно восстанавливаются по внутренним напряжениям вследствие теоремы 1 . В случае б) подграф̆ $\mathbf{G}_{1}$ содержит единственный закрепленньй шарнир $\mathbf{p}_{m+1}$ ЗШС. Будем обозначать подшарнирник, отвечающий подграфу $\mathbf{G}_{i}$, также $\mathbf{G}_{i}$. Структура нашего разбиения такова, что усилия от одного подшарнирника $\mathbf{G}_{i}$ не могут передаваться к другому подшарнирнику $\mathbf{G}_{j}$, поэтому напряжения в подшарнирнике $\mathbf{G}_{1}$ взаимно уравновешиваются. Применяя лемму 5 , нетрудно доказать, что если задано внутреннее напряжение подшарнирника $\mathbf{G}_{1}$, то положения свободных шарниров подшарнирника $\mathbf{G}_{1}$ образуют однопараметрическое семейство. (Подшарнирник $\mathbf{G}_{1}$ можно линейным преобразованием растягивать от точки $x_{m+1}$, при этом его внутреннее напряжение не изменяется.) Добавление каждого следующего подшарнирника $\mathbf{G}_{i}$ увеличивает число параметров неопределенности на 1 . При этом дело обстоит в точности так же, как и с подшарнирником $\mathbf{G}_{1}$ в случае а).

\section{§ 3. Шарнирники в $\mathbb{R}^{d}$ при $d>1$}

Сначала рассмотрим два примера в плоскости. Первый - это нетривиальньй пример такой ЗШС, что все несократимые полностью напрягаемые шарнирники, отвечающие ей, восстановимы. Во втором примере проявляются коренные отли- 

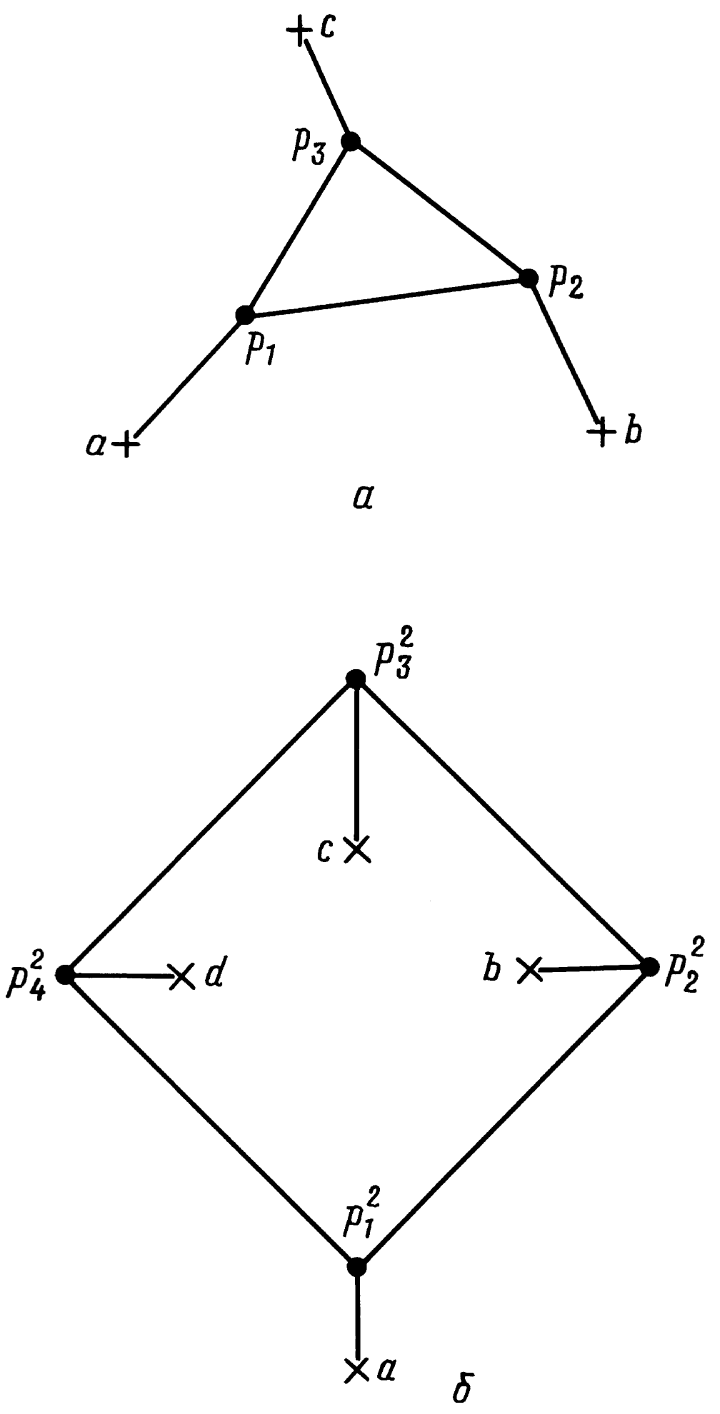

Рис. 3

чия плоского случая от одномерного, а также сложность задачи восстановления шарнирника уже в плоском случае.

ПримеР 1. У ЗШС, изображенной на рис. 3 , а, закрепленные шарниры $a, b, c$ не лежат на одной прямой. Возьмем такую декартову прямоугольную систему координат в плоскости, чтобы $a=(0,0), b=(1,0), c=\left(x_{c}, y_{c}\right)$; по условию $y_{c} \neq 0$. Обозначая координаты пространства внутренних напряжений $\left(\omega_{1 a}, \omega_{12}, \omega_{13}\right.$, $\left.\omega_{2 b}, \omega_{23}, \omega_{3 c}\right)$, можно записать

$$
\operatorname{det} \Omega(\omega)=\left|\begin{array}{ccc}
\omega_{1 a}+\omega_{12}+\omega_{13} & -\omega_{12} & -\omega_{13} \\
-\omega_{21} & \omega_{2 b}+\omega_{21}+\omega_{23} & -\omega_{23} \\
-\omega_{31} & -\omega_{32} & \omega_{3 c}+\omega_{31}+\omega_{32}
\end{array}\right|
$$


Необходимым условием невосстановимости шарнирника $\mathbf{p}$ с данной ЗШС является равенство нулю этого определителя при произвольном $\omega \in W(\mathbf{p})$. Мы получим условия на координаты $\left(x_{i}, y_{i}\right)$ свободных шарниров $\mathbf{p}_{i}, 1 \leqslant i \leqslant 3$, необходимые для равенства $\operatorname{det} \Omega(\omega)=0$. Из первого уравнения векторной системы $(3)$ для нашей ЗШС

$$
\omega_{12}\left(\mathbf{p}_{1}-\mathbf{p}_{2}\right)+\omega_{13}\left(\mathbf{p}_{1}-\mathbf{p}_{3}\right)=-\omega_{1 a}\left(\mathbf{p}_{1}-\mathbf{p}_{a}\right)=-\omega_{1 a} \mathbf{p}_{1}
$$

выразим напряжения $\omega_{12}$ и $\omega_{13}$ через $\omega_{1 a}$ :

$$
\omega_{12}=\frac{\omega_{1 a} \Delta_{13}}{\Delta_{12}-\Delta_{13}+\Delta_{23}}, \quad \omega_{13}=-\frac{\omega_{1 a} \Delta_{12}}{\Delta_{12}-\Delta_{13}+\Delta_{23}}, \quad \text { где } \quad \Delta_{i j}=\left|\begin{array}{ll}
x_{i} & x_{j} \\
y_{i} & y_{j}
\end{array}\right| .
$$

Точно так же из второго уравнения системы (3) найдем

а из третьего

$$
\omega_{21}=-\frac{\omega_{2 b}\left(\Delta_{23}+y_{2}-y_{3}\right)}{\Delta_{12}-\Delta_{13}+\Delta_{23}}, \quad \omega_{23}=-\frac{\omega_{2 b}\left(\Delta_{13}+y_{1}-y_{2}\right)}{\Delta_{12}-\Delta_{13}+\Delta_{23}}
$$

$$
\omega_{31}=-\frac{\omega_{3 c}\left(\Delta_{23}+\Delta_{3 c}-\Delta_{2 c}\right)}{\Delta_{12}-\Delta_{13}+\Delta_{23}}, \quad \omega_{32}=\frac{\omega_{3 c}\left(\Delta_{13}+\Delta_{3 c}-\Delta_{1 c}\right)}{\Delta_{12}-\Delta_{13}+\Delta_{23}} .
$$

Подставляя эти выражения в (8), получим

$$
\begin{aligned}
\operatorname{det} \Omega(\omega)= & -\frac{\omega_{1 a} \omega_{2 b} \omega_{3 c}}{\left(\Delta_{12}-\Delta_{13}+\Delta_{23}\right)^{3}} \\
& \times\left|\begin{array}{ccc}
\Delta_{23} & -\Delta_{13} & \Delta_{12} \\
-\Delta_{23}-y_{2}+y_{3} & \Delta_{13}+y_{1}-y_{3} & -\Delta_{12}-y_{1}+y_{2} \\
\Delta_{23}+\Delta_{3 c}-\Delta_{2 c} & -\Delta_{13}-\Delta_{3 c}-\Delta_{1 c} & \Delta_{12}+\Delta_{2 c}-\Delta_{1 c}
\end{array}\right| \\
= & \frac{\omega_{1 a} \omega_{2 b} \omega_{3 c}}{\left(\Delta_{12}-\Delta_{13}+\Delta_{23}\right)^{3}}\left|\begin{array}{ccc}
\Delta_{12}-\Delta_{13}+\Delta_{23} & -\Delta_{13} & \Delta_{12} \\
0 & y_{1}-y_{3} & -y_{1}+y_{2} \\
0 & \Delta_{3 c}-\Delta_{1 c} & \Delta_{2 c}-\Delta_{1 c}
\end{array}\right| \\
= & \frac{\omega_{1 a} \omega_{2 b} \omega_{3 c}}{\Delta_{12}-\Delta_{13}+\Delta_{23}} \cdot y_{c} .
\end{aligned}
$$

Таким образом, равенство $\operatorname{det} \Omega(\omega)=0$ может иметь место, если либо вьполнено хотя бы одно из равенств $\omega_{1 a}=0, \omega_{2 b}=0, \omega_{3 c}=0$, либо

$$
\Delta_{12}-\Delta_{13}+\Delta_{23}=\left|\begin{array}{ccc}
1 & 1 & 1 \\
x_{1} & x_{2} & x_{3} \\
y_{1} & y_{2} & y_{3}
\end{array}\right|=\left|\begin{array}{cc}
x_{2}-x_{1} & x_{3}-x_{1} \\
y_{2}-y_{1} & y_{3}-y_{1}
\end{array}\right|=0 .
$$

Первая из возможностей противоречит полной напрягаемости нашего шарнирника. Вторая возможность означает принадлежность всех трех свободных шарниров одной прямой. Поскольку все три закрепленных шарнира не лежат на одной прямой, то вторая возможность также противоречит полной напрягаемости шарнирника. Этим доказано, что всякий полностью напрягаемьй несократимый шарнирник с рассматриваемой ЗШС восстанавливается по своему пространству напряжений. 
ПримеР 2. У ЗШС, изображенной на рис. 3 , б, закрепленные шарниры $a, b, c, d$ находятся в точках $(0,-2),(1 / 2,0),(0,2 / 7),(-1 / 2,0)$ соответственно. Свободные шарниры шарнирника $\mathbf{p}^{1}$ имеют координаты $\mathbf{p}_{1}^{1}=(0,-3), \mathbf{p}_{2}^{1}=(3,0), \mathbf{p}_{3}^{1}=(0,3)$, $\mathbf{p}_{4}^{1}=(-3,0)$. При этом матрица

$$
\left|\begin{array}{cccccccc}
-3 & 0 & 0 & 3 & 0 & 0 & 0 & 0 \\
-3 & 0 & 0 & -3 & -1 & 0 & 0 & 0 \\
3 & 3 & 0 & 0 & 0 & -2.5 & 0 & 0 \\
3 & -3 & 0 & 0 & 0 & 0 & 0 & 0 \\
0 & 3 & -3 & 0 & 0 & 0 & 0 & 0 \\
0 & 3 & 3 & 0 & 0 & 0 & 19 / 7 & 0 \\
0 & 0 & -3 & -3 & 0 & 0 & 0 & -2.5 \\
0 & 0 & -3 & 3 & 0 & 0 & 0 & 0
\end{array}\right|
$$

системы уравнений (3) относительно неизвестных $\omega_{i j}$ имеет ранг, равный 7 . Следовательно, шарнирник $\mathbf{p}^{1}$ обладает одномерньм пространством внутренних напряжений. Как легко проверить, оно порождается вектором $\omega^{1}$ с координатами $\omega_{12}=\omega_{23}=\omega_{34}=\omega_{41}=1, \omega_{1 a}=-6, \omega_{2 b}=-2.4, \omega_{3 c}=-42 / 19, \omega_{4 d}=-2.4$. В этом случае

$$
\operatorname{det} \Omega\left(\omega^{1}\right)=\left|\begin{array}{cccc}
-4 & -1 & 0 & -1 \\
-1 & -0.4 & -1 & 0 \\
0 & -1 & -4 / 19 & -1 \\
-1 & 0 & -1 & -0.4
\end{array}\right|=0.4 \cdot 0.6 \cdot 4 / 19 \neq 0 .
$$

Таким образом, шарнирник $\mathbf{p}^{1}$ восстановим по своему пространству напряжений.

Шарнирник $\mathbf{p}^{2}$ с той же ЗШС и свободными шарнирами $\mathbf{p}_{1}^{2}=(0,-1), \mathbf{p}_{2}^{2}=$ $(1,0), \mathbf{p}_{3}^{2}=(0,1), \mathbf{p}_{4}^{2}=(-1,0)$ также имеет одномерное пространство внутренних напряжений $W\left(\mathbf{p}^{2}\right)$. Оно порождается вектором $\omega^{2}$ с координатами $\omega_{12}=\omega_{23}=$ $\omega_{34}=\omega_{41}=1, \omega_{1 a}=2, \omega_{2 b}=\omega_{4 d}=-4, \omega_{3 c}=-14 / 5$. В этом случае

$$
\operatorname{det} \Omega\left(\omega^{2}\right)=\left|\begin{array}{cccc}
4 & -1 & 0 & -1 \\
-1 & -2 & -1 & 0 \\
0 & -1 & -4 / 5 & -1 \\
-1 & 0 & -1 & -2
\end{array}\right|=0
$$

и потому шарнирник $\mathbf{p}^{2}$ невосстановим по пространству $W\left(\mathbf{p}^{2}\right)$.

Докажем теперь теорему, доставляющую достаточные условия для невосстановимости шарнирника по его пространству внутренних напряжений. Рассмотрим шарнирник $\mathbf{p}$ в $\mathbb{R}^{d}(d \geqslant 1)$ с ЗШС $\mathfrak{Z}$ и ненулевым пространством $W$ напряжений. 
ТЕОРЕМА 2. Пусть множество $V$ шарниров шарнирника $\mathbf{p}$ так распадается на три непересекающиеся части $V_{1}, V_{2}, V_{3}$, әде $V_{1}$ - непустое множество, состоящее лишь из свободных шарниров, $V_{2}$ - непустое множество, могущее содержать как свободные, так и закрепленные шарниры, $V_{3}$ - возможнно, пустое множсество, могущее содержать свободные и закрепленнье шарниры, что не существует рычагов, соединяющих шарниры из множества $V_{1}$ с шарнирами множества $V_{3}$. Все шарниры из $V_{2}$ лежат в $k$-мерном $(k<d)$ линейном многообразии $L \subset \mathbb{R}^{d}, u$, кроме того, все равнодействующие сил, приложенных от шарниров множсества $V_{1} \kappa$ шарнирам из $V_{2}$, при любом напряжении $\omega \in W$ лежсат в многообразии L. Тогда для произвольного аффинного преобразования $\mathbb{A}$ пространства $\mathbb{R}^{d}$, оставляющего все точки многообразия L на месте, шарнирник $\mathbf{p}^{\prime}$ с шарнирами $\mathbf{p}_{i}^{\prime}=\mathbf{p}_{i}$ при $v_{i} \in V_{2}, V_{3}$ $u \mathbf{p}_{i}^{\prime}=\mathbb{A}_{i}$ nри $v_{i} \in V_{1}$ и ЗШС $\mathfrak{Z}$ имеет то же самое подпространство $W$ внутренних напряжений, что и шарнирник $\mathbf{p}$.

ДокаЗАТЕЛЬСтво. Разобьем систему уравнений (3) относительно положений свободных шарниров для ЗШС $\mathfrak{Z}$ на три части:

$$
\begin{array}{ll}
\sum_{j} \omega_{i j}\left(\mathbf{p}_{j}-\mathbf{p}_{i}\right)=\mathbf{0}, & v_{i} \in V_{1}, \quad v_{j} \in V_{1}, V_{2}, \\
\sum_{j} \omega_{i j}\left(\mathbf{p}_{j}-\mathbf{p}_{i}\right)=\mathbf{0}, & v_{i} \in V_{2}, \quad v_{j} \in V, \\
\sum_{j} \omega_{i j}\left(\mathbf{p}_{j}-\mathbf{p}_{i}\right)=\mathbf{0}, & v_{i} \in V_{3},
\end{array}
$$

Здесь суммирование проводится по всем шарнирам $v_{j}$, смежным с шарниром $v_{i}$ и удовлетворяющим выписанным условиям, а вектор $\omega \in W$ считается произвольным. Эта система удовлетворяется набором $\left(\mathbf{p}_{1}, \ldots, \mathbf{p}_{m}\right)$ положений свободных шарниров шарнирника $\mathbf{p}$. Покажем, что ей удовлетворяет и шарнирник $\mathbf{p}^{\prime}=$ $\left(\mathbf{p}_{1}^{\prime}, \ldots, \mathbf{p}_{m}^{\prime}\right)$. Поскольку $\mathbf{p}_{i}^{\prime}=\mathbf{p}_{i}$ при $v_{i} \in V_{2}, V_{3}$, то справедливость равенств (в) очевидна.

Далее, выбирая начало координат в точке многообразия $L$, имеем

$$
\mathbb{A}(\alpha \mathbf{y}+\beta \mathbf{z})=\alpha \mathbb{A}(\mathbf{y})+\beta \mathbb{A}(\mathbf{z})
$$

для произвольных векторов $\mathbf{y}, \mathbf{z} \in \mathbb{R}^{d}$ и $\alpha, \beta \in \mathbb{R}$, а также $\mathbb{A}(\mathbf{x})=\mathbf{x}$ для $\mathbf{x} \in L$. $\mathrm{B}$ частности, $\mathbb{A}(\mathbf{0})=\mathbf{0}$. Следующая выкладка устанавливает справедливость равенств (а) для шарнирника $\mathbf{p}^{\prime}$ :

$$
\sum_{j} \omega_{i j}\left(\mathbf{p}_{j}^{\prime}-\mathbf{p}_{i}^{\prime}\right)=\sum_{j} \omega_{i j}\left(\mathbb{A} \mathbf{p}_{j}-\mathbb{A} \mathbf{p}_{i}\right)=\mathbb{A}\left(\sum_{j} \omega_{i j}\left(\mathbf{p}_{j}-\mathbf{p}_{i}\right)\right)=\mathbb{A}(\mathbf{0})=\mathbf{0}
$$


Уравнения (б) перепишем в следуюшем виде:

$$
\sum_{v_{j} \in V_{1}} \omega_{i j}\left(\mathbf{p}_{j}-\mathbf{p}_{i}\right)=-\sum_{v_{j} \in V_{2}, V_{3}} \omega_{i j}\left(\mathbf{p}_{j}-\mathbf{p}_{i}\right) .
$$

По условию теоремы $\sum_{v_{j} \in V_{1}} \omega_{i j}\left(\mathbf{p}_{j}-\mathbf{p}_{i}\right) \in L$ при $v_{i} \in V_{2}$, а следовательно,

$$
\begin{aligned}
\sum_{v_{j} \in V_{1}} \omega_{i j}\left(\mathbf{p}_{j}^{\prime}-\mathbf{p}_{i}^{\prime}\right) & =\sum_{v_{j} \in V_{1}} \omega_{i j}\left(\mathbb{A} \mathbf{p}_{j}-\mathbb{A} \mathbf{p}_{i}\right) \\
& =\mathbb{A}\left(\sum_{v_{j} \in V_{1}} \omega_{i j}\left(\mathbf{p}_{j}-\mathbf{p}_{i}\right)\right)=\sum_{v_{j} \in V_{1}} \omega_{i j}\left(\mathbf{p}_{j}-\mathbf{p}_{i}\right) .
\end{aligned}
$$

Подставляя радиус-векторы шарниров шарнирника $\mathbf{p}^{\prime}$ в правую часть уравнений $\left(\sigma^{\prime}\right)$, также получим

$$
-\sum_{v_{j} \in V_{2}, V_{3}} \omega_{i j}\left(\mathbf{p}_{j}^{\prime}-\mathbf{p}_{i}^{\prime}\right)=-\sum_{v_{j} \in V_{2}, V_{3}} \omega_{i j}\left(\mathbf{p}_{j}-\mathbf{p}_{i}\right) .
$$

Таким образом, шарнирник $\mathbf{p}^{\prime}$ обладает внутренним напряжением $\omega$.

Мы доказали, что каждое внутреннее напряжение шарнирника $\mathbf{p}$ является и напряжением шарнирника $\mathbf{p}^{\prime}$. C другой стороны, поскольку шарнирник $\mathbf{p}$ можно получить из шарнирника $\mathbf{p}^{\prime}$ точно таким же способом, используя преобразование $\mathbb{A}^{-1}$, то верно и обратное. Теорема доказана.

СлЕДСТВИЕ 1. Если к условиям теоремы 2 добавить еще одно условие, а именно, что не все иарниры множества $V_{1}$ лежат в многообразии L, то шарнирник $\mathbf{p}$ заведомо не будет восстанавливаться по своему пространству внутренних напряжений.

Сейчас мы получим еще одно следствие теоремы 2 , свободное от каких-либо предположений о равнодействуюших внутренних сил. Известно (см. [4], [1]), что если выбрать произвольную совокупность $\mathscr{V}$ шарниров внутренне напряженного шарнирника p, то силы, приложенные со стороны этих выбранных шарниров к остальным (из $V \backslash \mathscr{V}$ ) шарнирам шарнирника, составляют уравновешенную систему. Система сил называется уравновешенной, если сумма сил этой системы, а также их момент нулевые.

УТВЕРЖДДЕНИЕ 3 . Пусть $к$ точкам $p_{0}, p_{1}, \ldots, p_{k}$, находящимся в общем положении в $\mathbb{R}^{d}$, приложены силь $F_{0}, F_{1}, \ldots, F_{k}$ соответственно, составляющие уравновешенную систему. Тогда если $k<d$, все то векторы $F_{0}, F_{1}, \ldots$, $F_{k}$ лежат в аффинной оболочке $L^{k}$ точек $p_{0}, p_{1}, \ldots, p_{k}$. 
ДокАЗАТЕльство. В самом деле, выберем в $\mathbb{R}^{d}$ декартову, не обязательно прямоугольную систему координат с началом в точке $p_{0}$ и первыми $k$ базисными векторами $e_{i}=p_{0} p_{i}, 1 \leqslant i \leqslant k$. Необходимым условием равенства нулю момента системы сил $F_{0}, F_{1}, \ldots, F_{k}$ в случае произвольной размерности является равенство нулевому тензору суммы бивекторов $F_{j} \wedge p_{j}$. Полагая во введенной системе координат

$$
F_{j}=\sum_{i=1}^{d} f_{j}^{i} e_{i}
$$

запишем это условие:

$$
\sum_{j=0}^{k} F_{j} \wedge p_{j}=\sum_{i, j=1}^{k} f_{j}^{i} e_{i} \wedge e_{j}+\sum_{\substack{j=1 \\ i>k}} f_{j}^{i} e_{i} \wedge e_{j}=0
$$

Поскольку каждый из бивекторов $e_{i} \wedge e_{j}$ при $j \leqslant k, i>k$ встречается лишш в последней сумме и лишь единожды, то $f_{j}^{i}=0$ при $i>k$. Это и означает, что все векторы $F_{0}, F_{1}, \ldots, F_{k}$ лежат в $L^{k}$.

СЛЕДСТВИЕ 2. Пусть множество $V$ шарниров шарнирника $\mathbf{p}$ так распадается на три непересекающиеся части $V_{1}, V_{2}, V_{3}$, дде $V_{1}$ - непустое множество, состоящее лишь из свободных шарниров, $V_{2}$ - непустое множество, содержсащее $k \leqslant d$ шарниров, среди которых могут быть как свободные так u закрепленные, $V_{3}$ - возможсно, пустое множество, могущее содержать свободные и закрепленные шарниры, что не существует рычагов, соединяющих шарниры из множества $V_{1}$ с шарнирами множества $V_{3}$. И пусть все $k$ шарниров множества $V_{2}$ находятся в общем положении, а их аффинная оболочка не содержит всех шарниров множества $V_{1}$. Тогда шарнирник $\mathbf{p}$ невосстановим по своему пространству напряжений.

\section{§4. Образ рычажного отображения и шарнирники, допускающие внутренние напряжения}

Задание ЗШС $\mathfrak{Z}$ в $\mathbb{R}^{d}$ с $m$ свободными шарнирами и $r$ рычагами определяет рычажное отображение [1] $\mathbf{F}: \mathbb{R}^{d m} \rightarrow \mathbb{R}^{r}$ формулами $d_{i j}=\left(\mathbf{p}_{i}-\mathbf{p}_{j}\right)^{2}$, где декартова координата $d_{i j}$ пространства $\mathbb{R}^{r}$ имеет смысл квадрата длины рычага $\mathbf{p}_{i} \mathbf{p}_{j}$. Образ $\mathscr{C}=\mathbf{F}\left(\mathbb{R}^{d m}\right)$ этого отображения, очевидно, лежит в неотрицательном октанте $Q: d_{i j} \geqslant 0$ стандартной координатной системы евклидова пространства $\mathbb{R}^{r}$. Строение множества $\mathscr{C}$ определяет общие геометрические свойства совокупности шарнирников, имеюших ЗШС $\mathfrak{3}$. Некоторые сведения о строении этого множества можно найти в статье [1]. Здесь будет исследована связь между граничными точками множества $\mathscr{C}$ и шарнирниками, допускаюшими внутренние напряжения.

Пусть $\omega=\left\{\omega_{i j}\right\}$ - допустимое для ЗШС $\mathfrak{Z}$ напряжение. Множество шарнирников, допускающих это напряжение, определяется как решение соответствующей 
системы уравнений (4) и представляет собой либо точку, либо линейное многообразие $L_{\omega} \subset \mathbb{R}^{d m}$ размерности, не меньшей 1 .

Теорема 3. Образ $\mathbf{F}\left(L_{\omega}\right)$ многообразия $L_{\omega}$ при рычажном отображении связен, неограничен и лежит в плоскости пространства $\mathbb{R}^{r}$, задающейся уравнением $\sum_{v_{i} v_{j} \in E} \omega_{i j} d_{i j}=c$, где $c$ - const (здесь $E-$ множество рычагов ЗШIC 3$).$

ДоказАтеЛЬСтво. Связность множества $\mathbf{F}\left(L_{\omega}\right)$ вытекает из непрерывности отображения $\mathbf{F}$, неограниченность же следует из леммы 1 работы [1]. Для доказательства последнего из утверждений теоремы рассмотрим однопараметрическое линейное семейство шарнирников $\mathbf{p}(t)=\mathbf{a}+\mathbf{b} t \in L_{\omega}, 1<t<\infty$. Вычислим производную функции

$$
\Phi(t)=\sum_{v_{i} v_{j} \in E} \omega_{i j} d_{i j}(\mathbf{p}(t))
$$

в произвольной точке этого семейства:

$$
\begin{aligned}
\frac{d \Phi}{d t} & =2 \sum_{v_{i} v_{j} \in E} \omega_{i j}\left(\mathbf{p}_{i}-\mathbf{p}_{j}, \dot{\mathbf{p}}_{i}-\dot{\mathbf{p}}_{j}\right) \\
& =2 \sum_{v_{i} v_{j} \in E}\left[\omega_{i j}\left(\mathbf{p}_{i}-\mathbf{p}_{j}, \dot{\mathbf{p}}_{i}\right)+\omega_{i j}\left(\mathbf{p}_{j}-\mathbf{p}_{i}, \dot{\mathbf{p}}_{j}\right)\right] \\
& =2 \sum_{i=1}^{m}\left(\dot{\mathbf{p}}_{i}, \sum_{\substack{j \\
v_{i} v_{j} \in E}} \omega_{i j}\left(\mathbf{p}_{i}-\mathbf{p}_{j}\right)\right)=0 .
\end{aligned}
$$

Здесь мы воспользовались равенством $\dot{\mathbf{p}}_{i}=0$ производной по $t$ для закрепленных шарниров $\mathbf{p}_{i}$ и справедливостью условий (3) равновесия сил в каждом свободном шарнире для произвольного шарнирника из $L_{\omega}$. Поскольку $\frac{d \Phi}{d t}=0$ в каждой точке многообразия $L_{\omega}$, то

$$
\Phi=\sum_{v_{i} v_{j} \in E} \omega_{i j} d_{i j}=c, \quad \text { где } c-\text { const }
$$

на множестве $\mathbf{F}\left(L_{\omega}\right)$. Теорема доказана.

Касательным многообразием $T\left(\mathbf{p}^{0}\right)$ рычажного отображения $\mathbf{F}: \mathbb{R}^{d m} \rightarrow \mathbb{R}^{r}$ в точке $\mathbf{p}^{0} \in \mathbb{R}^{d m}$ назовем линейное многообразие пространства $\mathbb{R}^{r}$, состояшее из точек $\mathbf{d}=\mathbf{F}\left(\mathbf{p}^{0}\right)+d \mathbf{F} \cdot \mathbf{x}$, где $d \mathbf{F}$ - матрица дифференциала отображения $\mathbf{F}$ в точке $\mathbf{p}^{0}, \mathrm{a} \mathbf{x} \in \mathbb{R}^{d m}$.

ЛЕмма 6. Пусть $\mathbf{d}^{0}=\left\{d_{i j}^{0}\right\}=\mathbf{F}\left(\mathbf{p}^{0}\right) ;$ тогда касательное многообразие $T\left(\mathbf{p}^{0}\right)$ совпадает с пересечением $\Pi$ всех $(r-1)$-мерных плоскостей $\Pi_{\omega}$, задающихся уравнениями

$$
\sum_{v_{i} v_{j} \in E} \omega_{i j}\left(d_{i j}-d_{i j}^{0}\right)=0
$$


где наборы коэффичиентов $\omega_{i j}$ составляют напряжения иарнирника $\mathbf{p}^{0}$.

Действительно, матрица Якоби $d \mathbf{F}$ есть $(r \times d m)$-матрица, каждая строка которой отвечает рычагу шарнирника, а каждый столбец - координате одного из свободных шарниров. Если столбцы этой матрицы считать столбцами координат векторов в $\mathbb{R}^{r}$, то линейная оболочка $\mathscr{L}$ этих векторов совпадает с множеством векторов пространства $\mathbb{R}^{r}$, представимых в виде $d \mathbf{F} \cdot \mathbf{x}$, где вектор-столбец $\mathbf{x}$ принадлежит $\mathbb{R}^{d m}$. Наличие же у шарнирника $\mathbf{p}^{0}$ напряжения $\omega=\left\{\omega_{i j}\right\}$ означает линейную с коэффициентами $\omega_{i j}$ зависимость строк матрицы $d \mathbf{F}$. Иными словами, вектор $\omega \in \mathbb{R}^{r}$ в том и только том случае является напряжением шарнирника $\mathbf{p}^{0}$, когда он ортогонален каждому столбцу матрицы $d \mathbf{F}$. Таким образом, пространство напряжений шарнирника и пространство $\mathscr{L}$ являются взаимно ортогональными и взаимно дополнительными подпространствами пространства $\mathbb{R}^{r}$.

Если линейное многообразие П так перенести параллельно, чтобы оно прошло через начало координат, то оно также станет подпространством, ортогональным и дополнительным к пространству напряжений шарнирника $\mathbf{p}^{0}$. Итак, многообразия П и $T\left(\mathbf{p}^{0}\right)$ имеют одинаковую размерность, параллельны и проходят через одну точку $\mathbf{d}^{0}$, а следовательно, совпадают.

Мы называем $(r-1)$-мерную плоскость $\Pi \subset \mathbb{R}^{r}$ опорной $\kappa$ множеству $\mathscr{C}$ в точке $\mathbf{d}$ его границы, если $\mathbf{d} \in \Pi$ и множество $\mathscr{C}$ лежит в одном из замкнутых полупространств, ограниченных плоскостью П. Мы называем плоскость П локально опорной $\kappa$ множеству $\mathscr{C}$ в точке $\mathbf{d}$ его границы, если найдется такая окрестность $\mathscr{O}$ точки d, что плоскость П опорна к множеству $\mathscr{O} \cap \mathscr{C}$ в точке $\mathbf{d}$. Мы говорим, что плоскость П касается множества $\mathscr{A} \subset \mathbb{R}^{r}$ в его граничной точке $\mathbf{d}$, если предельное положение прямой (секущей) $\mathbf{d d}^{\prime}, \mathbf{d}^{\prime} \in \partial \mathscr{A}$, при произвольном стремлении $\mathbf{d}^{\prime}$ к $\mathbf{d}$, есть прямая, лежащая в плоскости П.

ЛЕмма 7. Если образ $\mathbf{F}(\mathbf{p})$ шарнирника $\mathbf{p}$ лежит на граниче ӘС્ множества $\mathscr{C}$, то шарнирник $\mathbf{p}$ допускает внутреннее напряжение. Более того, если плоскость $\Pi_{\omega}: \sum_{v_{i} v_{j} \in E} \omega_{i j} d_{i j}=c$, әде $c$ - const, локально опорна либо касается $\mathscr{C}$ в точке $\mathbf{d}^{0} \in \partial \mathscr{C}$, то множество $\mathbf{F}^{-1}\left(\mathbf{d}^{0}\right)$ непусто и каждый шарнирник из этого множества допускает напряжение $\omega=\left\{\omega_{i j}\right\}$.

ДокАЗАТЕЛЬСТво. Действительно, предположим, что шарнирник $\mathbf{p}$ не допускает внутренних напряжений. Тогда строки матрицы Якоби $d \mathbf{F}(\mathbf{p})$ линейно независимы. Отсюда следует, что $r \leqslant d m$, а у точки $\mathbf{F}(\mathbf{p}) \in \mathscr{C}$ найдется окрестность $\mathscr{O} \subset \mathbb{R}^{r}$, каждая точка $\mathbf{d}$ которой имеет непустой прообраз $\mathbf{F}^{-1}(\mathbf{d})[5]$.

В силу замкнутости множества $\mathscr{C}[1]$ из $\mathbf{d} \in \partial \mathscr{C}$ вытекает $\mathbf{d} \in \mathscr{C}$, и потому $\mathbf{F}^{-1}(\mathbf{d})$ непусто. Пусть $\mathbf{p}^{k} \in \mathbf{F}^{-1}(\mathbf{d})$ и в точке $\mathbf{d}$ имеется касательная либо локально опорная к $\mathscr{C}$ плоскость $\Pi_{\omega}$. Тогда касательное многообразие $T\left(\mathbf{p}^{k}\right)$ очевидным образом лежит в плоскости $\Pi_{\omega}$. Отсюда следует допустимость напряжения $\omega=\left\{\omega_{i j}\right\}$ для шарнирника $\mathbf{p}^{k}$.

Из последней леммы и теоремы 3 вытекает, что касательная либо локально опорная к $\mathscr{C}$ плоскость $\Pi_{\omega}$ содержит образ $\mathbf{F}\left(\mathscr{L}_{\omega}\right)$ множества $\mathscr{L}_{\omega}$ шарнирников, 
допускающих напряжение $\omega$. Отсюда следует интересное и, на мой взгляд, важное геометрическое свойство множества $\mathscr{C}$.

УТВЕРЖДЕНИЕ 4. Множество $\mathscr{C}$ имеет не более одной локально опорной либо касательной плоскости $\Pi_{\omega}$ произвольно заданного направления $\omega$.

В этом утверждении направления $\omega$, естественно, рассматриваются как точки проективного пространства $\mathbb{R} \mathbb{P}^{r-1}$.

ЛЕмма 8. Пусть в точке $\mathbf{d} \in \partial \mathscr{C}$ имеется касательная либо опорная $\kappa \mathscr{C}$ плоскость $\Pi_{\omega}$ и пересечение $\Pi_{\omega} \cap \mathscr{C}$ ограничено. Тогда $\mathbf{d}$ - однократная точка отображсени $\mathbf{F}$ и имеется лишь один шарнирник, допускающий напряэсение $\omega$.

ДокАЗАТЕЛЬСтво. Действительно, предположим, что $\mathbf{F}^{-1}(\mathbf{d})$ содержит две различных точки: $\mathbf{p}_{1}$ и $\mathbf{p}_{2}$. Вследствие леммы 7 оба шарнирника, $\mathbf{p}_{1}$ и $\mathbf{p}_{2}$, допускают внутреннее напряжение $\omega$, а значит, имеется не менее чем одномерное многообразие $L_{\omega}$ шарнирников, допускающих напряжение $\omega$. Но тогда образ $\mathbf{F}\left(L_{\omega}\right)$ по теореме 3 есть неограниченное множество, лежащее в плоскости $\Pi_{\omega}$. Это противоречит условию леммы.

Р. Коннелли рассматривал (см. [6]) обобшения шарнирных конструкций, могушие содержать кроме рычагов еше и нерастяжимые нити, связываюшие шарниры, а также распорки, не позволяющие отдельным парам шарниров сближаться более определенного предела. Эти обобщения были названы "tensegrety frameworks" (что можно перевести как напряженно-связанные конструкции), а в случае конструкций, состоящих из одних лишь нитей, паутинами - "spider werbs". Коннелли была установлена (см. [2]) восстановимость паутин по их напряжениям $\left\{\omega_{i j}\right\}$, $\omega_{i j}>0$. Назовем паутинным шарнирник, допускающий набор внутренних напряжений одного знака. Из результата Коннелли следует восстановимость паутинных шарнирников по их напряжениям. Мы установим это иным способом.

ТЕОРЕМА 4. Какова бы ни была ЗШС $\mathfrak{Z}$, каждое внутреннее напряжение $\omega=\left\{\omega_{i j}\right\}, \omega_{i j} \geqslant 0$, является допустимым для нее. Если жсе все $\omega_{i j}>0$, то по такому напряжению шарнирник $\mathbf{p}$, возможсо, сократимый, восстановим однозначно. Более того, в этом случае не существует других иарнирников с ЗШС $\mathfrak{Z}$ и тем же самым набором длин ребер, что и у р.

ДокАЗАТЕЛЬСтво. Поскольку множество $\mathscr{C}$ лежит в неотрицательном октанте $Q \subset \mathbb{R}^{r}$, то каков бы ни был вектор $\omega=\left\{\omega_{i j}\right\} \in \mathbb{R}^{r}, \omega_{i j} \geqslant 0$, у множества $\mathscr{C}$ непременно найдется опорная плоскость $\Pi_{\omega}$ с нормальным вектором $\omega$.

Если все $\omega_{i j}>0$, то пересечение $\Pi_{\omega} \cap Q$ ограничено, и в силу замкнутости множества $\mathscr{C}$ оно имеет общую точку $\mathbf{d}$ с плоскостью $\Pi_{\omega}$. Вследствие леммы 7 у точки $\mathbf{d}$ имеется прообраз $\mathbf{p}$, представляюший собой шарнирник, допускающий напряжение $\omega$. Таким образом, напряжение $\omega$ является допустимым для ЗШС 3. Если все $\omega_{i j}>0$, то пересечение $\Pi_{\omega} \cap \mathscr{C}$ ограничено. Вследствие леммы 8 
это возможно лишш в случае, когда множество $\mathscr{L}_{\omega}$ шарнирников, допускаюших напряжение $\omega$, состоит из одного лишь шарнирника $\mathbf{p}=\mathbf{F}^{-1}(\mathbf{d})$.

Остается доказать, что и в случае $\omega_{i j} \geqslant 0$ опорная плоскость $\Pi_{\omega}$ пересекается с множеством $\mathscr{C}$. Пусть набор $\mathscr{E}$ положительно напряженных рычагов в этом случае порождает граф $\mathscr{G}$, являюшийся подграфом графа $G$ ЗШС $\mathfrak{Z}$. Пусть $\mathbb{R}^{q}-$ координатное подпространство пространства $\mathbb{R}^{r}$, отвечающее рычагам из $\mathscr{E}$, а $\pi \mathscr{C}$ - ортогональная проекция множества $\mathscr{C}$ на $\mathbb{R}^{q}$. Нам достаточно установить замкнутость множества $\pi \mathscr{C}$.

Рассмотрим произвольную сходящуюся последовательность точек $\mathbf{d}_{i} \in \pi \mathscr{C}$ и докажем, что ее предельная точка $\mathbf{d}$ принадлежит $\pi \mathscr{C}$. Для этого выберем последовательность шарнирников $\mathbf{p}^{i}$ таких, что $\pi \mathbf{F}\left(\mathbf{p}^{i}\right)=\mathbf{d}_{i}$, следуюшим образом. Заметим, что граф $\mathscr{G}$ может состоять из нескольких компонент связности, не каждая из которых, однако, содержит закрепленные шарниры. Мы выберем последовательность $\left\{\mathbf{p}^{i}\right\}$ так, чтобы в каждой компоненте связности был фиксированный в пространстве шарнир, выбранный не зависящим от номера $i$ образом. Тогда последовательность шарниров из каждой компоненты связности ограничена и, следовательно, сушествует такое число $M$, что для каждого шарнира $\mathbf{p}_{k}^{i}$ шарнирника $\mathbf{p}^{i}$, принадлежащего графу $\mathscr{G},\left|\mathbf{p}_{k}^{i}\right|<M$ при $i \geqslant 1$. Поскольку $\pi \mathbf{F}\left(\mathbf{p}^{i}\right)$ не зависит от шарниров $\mathbf{p}_{k}^{i} \notin \mathscr{G}$, то их тоже можно выбрать так, чтобы было $\left|\mathbf{p}_{k}^{i}\right|<M$. Таким образом, последовательность $\left\{\mathbf{p}^{i}\right\}$ ограничена, а следовательно, она содержит сходящуюся к некоторой точке $\mathbf{p} \subset \mathbb{R}^{r}$ подпоследовательность. Вследствие непрерывности рычажного отображения и проектирования $\pi \mathbf{F}(\mathbf{p})=\mathbf{d}$. Таким образом, множество $\mathscr{C}$ замкнуто.

Итак, мы попутно доказали следующую лемму.

ЛЕмма 9. Ортогональная проекция множсества $\mathscr{C}=\mathbf{F}\left(\mathbb{R}^{d m}\right)$ на произвольное координатное подпространство $\mathbb{R}^{k}$ стандартной координатной системы в $\mathbb{R}^{r}$ замкнута.

Заметим еще, что из теоремы 4 вытекает неравенство $\operatorname{det} \Omega(\omega) \neq 0$ для произвольного напряжения $\omega=\left\{\omega_{i j}\right\}, \omega_{i j}>0$.

\section{§5. Существование несократимых паутинных шарнирников}

Этот параграф почти целиком посвяшен доказательству следующей теоремы.

ТЕОрема 5. Приведенной ЗШС $\mathfrak{Z}$ в $\mathbb{R}^{d}, d \geqslant 1$, тогда и только тогда отвечает хотя бы один несократимый паутинный иарнирник, когда ее дополненный граф $G^{\prime}$ не содержит разделяющих вершин, а число ее закрепленных шарниров больие 1 .

ДокАЗАТЕЛЬСТво. НЕОБХоДимость. Действительно, заметим, что каждьй из свободных шарниров паутинного шарнирника должен лежать в выпуклой оболочке смежных с ним шарниров. В противном случае, как легко видеть, равновесие сил в нем невозможно. Если в графе $G^{\prime}$ имеется разделяюшая вершина, то 
он распадается на несколько неодносвязных подграф̆ов, среди которых непременно имеется подграф $G_{i}$, не содержаший закрепленных шарниров и содержаший лишь одну из разделяющих вершин исходного графа $G$. Если шарнирник несократим, то в его части, отвечающей подграфу $G_{i}$, обязательно найдется свободный шарнир, не лежащий внутри выпуклой оболочки смежных с ним шарниров.

ДостАточность. Доказательство достаточности намного более трудоемко. Для того чтобы его провести, мы сначала определенным образом построим последовательность $\mathscr{Z}_{1}, \mathscr{Z}_{2}, \ldots, \mathscr{Z}_{k}=\mathfrak{Z}$ шарнирных схем, содержаших те же самые закрепленные шарниры, что и ЗШС $\mathfrak{Z}$. Поскольку графы схем этой последовательности могут содержать кратные ребра (т.е. на самом деле быть мультиграфами) и изолированные закрепленные шарниры, то эти схемы оправданно назвать псевдо-ЗШС. Заметим, что множества свободных шарниров наших псевдо-ЗШС будут являться подмножествами множества свободных шарниров ЗШС $\mathfrak{Z}$, упорядоченными по включению. Затем мы установим, что каждой псевдо-ЗШС из построенной последовательности отвечает несократимый паутинный шарнирник.

Для построения указанной последовательности ЗШС нам понадобится

УТВЕРЖДЕНИЕ 5. Пусть связный граф $\mathscr{G}$ не имеет разделяющих вериин, а его подграф $\mathscr{G}^{\prime}$ содержит не менее двух вериин, но не содержит всех вершин графа $\mathscr{G}$. Тогда в графе $\mathscr{G}$ найдется простая (т.е. не содержащая повторяющ,хся вершин) иепь, все внутренние вершины которой не принадлежсат подграфу $\mathscr{G}^{\prime}$, а конечнье принадлежсат $\mathscr{G}^{\prime}$ и различны.

ДокАЗАТЕльСТво. Возьмем произвольную вершину $v$ граф̆а $\mathscr{G}$, не принадлежащую подграфу $\mathscr{G}^{\prime}$. В силу связности графф $\mathscr{G}$ ее можно соединить простыми цепями с любой из вершин подграфа $\mathscr{G}^{\prime}$. Рассмотрим подмножество $M$ множества всех таких цепей, состоящее из цепей, содержащих ровно одну вершину (конечную), принадлежашую $\mathscr{G}^{\prime}$. Если в множестве $M$ найдутся две цепи, имеющие различные конечные вершины $v_{1} \in \mathscr{G}^{\prime}$ и $v_{2} \in \mathscr{G}^{\prime}$, то из объединения этих двух цепей, очевидно, можно выделить простую цепь требуемого в условии теоремы вида. Покажем теперь, что такие две цепи всегда найдутся. Допустим противное: все цепи множества $M$ имеют общую конечную вершину $v_{1} \in \mathscr{G}^{\prime}$. Тогда вершина $v_{1}$ является разделяющей в графе $\mathscr{G}$. Действительно, вершины $v$ и $v_{2}$ лежат в различных компонентах графа, полученного удалением из $\mathscr{G}$ вершины $v_{1}$ вместе с инцидентными ей ребрами. Это противоречит условию.

Поскольку закрепленные шарниры каждой из псевдо-ЗШС последовательности $\mathscr{Z}_{1}, \mathscr{Z}_{2}, \ldots, \mathscr{Z}_{k}$ совпадают с закрепленными шарнирами $v_{m+1}, v_{m+2}, \ldots, v_{m+n}$ ЗШС $\mathfrak{Z}$, то достаточно описать построение последовательности $G_{1}, G_{2}, \ldots, G_{k}$ мультиграфов этих псевдо-ЗШС.

Выберем граф $G_{1}$ следуюшим образом. Пусть $G_{0}-$ подграф графа $G^{\prime}$, состояший из вершин $v_{m+1}, v_{m+2}, \ldots, v_{m+n}(n \geqslant 2)$ и соединяюших их ребер $v_{m+1} v_{m+2}$, $\ldots, v_{m+1} v_{m+n}$. Вследствие утверждения 5 в граффе $G^{\prime}$ найдется простая цепь $\coprod_{1}$, все внутренние вершины которой не принадлежат подграфу $G_{0}$, а конечные вер- 
шины различны и принадлежат $G_{0}$. Добавив к графу $G_{0}$ цепь $\mathbf{\beth}_{1}$, получим граф $G_{1}$.

Построение остальных мультиграфов последовательности основано на следующей лемме.

ЛЕмма 10. Последовательность мультиграфов можно выбрать так, чтобъ мультиграф $G_{i+1}, \quad 1 \leqslant i \leqslant k-1$, получался из мультиграфа $G_{i}$ путем одного из следующих двух действий:

1) добавление $\kappa G_{i}$ ребра;

2) подразбиение ребра мультиграфа $G_{i}$, т.е. удаление этого ребра и добавление новой вершины и двух ребер, соединяющих ее с вершинами удаленного ребра.

ДокАЗАТЕльство. Если граф $G_{1}$ содержит все вершины графа $G^{\prime}$, но не совпадает с ним, то каждый из последуюших графов последовательности получается добавлением ребра к предыдущему. Если же $G_{1}$ не содержит всех вершин графа $G^{\prime}$, то вследствие утверждения 5 в $G^{\prime}$ найдется простая цеп Ц, из вершин которой лишь две конечные, $v^{\prime}$ и $v^{\prime \prime}$, принадлежат графу $G_{1}$. Соединяя их ребром, получим, вообще говоря, мультиграф $G_{2}$. Подразбивая ребро $v^{\prime} v^{\prime \prime}$ мультиграфа $G_{2}$, получим граф $G_{3}$. Ясно, что, повторяя подразбиение ребер, можно восстановить цепь Ц и получить графф $G_{i_{1}}=G_{1} \cup$ Ц являюшийся подграфом графа $G^{\prime}$. Если граф $G_{i_{1}}$ не совпадает с $G^{\prime}$, то можно продолжить построение последовательности мультиграфов описанным способом, лишь роль графа $G_{1}$ перейдет к графу $G_{i_{1}}$. Таким образом, мы дойдем до графа $G_{i_{s}}$, содержащего все вершины графа $G^{\prime}$. Останется добавить к графу $G_{i_{s}}$ некоторое количество ребер.

Перейдем к доказательству существования последовательности несократимых паутинных шарнирников.

Для построения несократимого паутинного шарнирника, отвечающего псевдоЗШС $\mathscr{Z}_{1}$, достаточно все шарниры цепи Ц 1 взять лежащими последовательно на отрезке прямой, соединяющем два конечных закрепленных шарнира этой цепи, и несовпадаюшими друг с другом. Возможность построения остальных шарнирников последовательности вытекает из следуюшей леммы.

ЛЕмма 11. Если псевдо-ЗШС $\mathscr{Z}_{\eta}, 1 \leqslant \eta \leqslant k-1$, отвечает несократимый паутинный шарнирник, то и псевдо-ЗШС $\mathscr{Z}_{\eta+1}$ также отвечает несократимый паутинньй шарнирник.

ДокАЗАТЕЛЬСтво. Пусть псевдо-ЗШС $\mathscr{Z}_{\eta}$ отвечает несократимый паутинный шарнирник $\mathbf{p}$, а граф $G_{\eta+1}$ получается подразбиением ребра мультиграфа $G_{\eta}$. Тогда несократимый паутинный шарнирник $\mathbf{p}^{\prime}$, отвечающий $\mathscr{Z}_{\eta+1}$, получим, помещая вновь вводимый шарнир внутрь рычага шарнирника $\mathbf{p}$, отвечающего подразбиваемому ребру.

Если мультиграф̆ $G_{\eta+1}$ получается из графа $G_{\eta}$ добавлением ребра, соединяюшего смежные вершины графа $G_{\eta}$, то шарнирник $\mathbf{p}^{\prime}$ можно взять с теми же положениями шарниров, что и у шарнирника р. При этом можно считать, что вместо 
одного положительно напряженного рычага в $\mathbf{p}$ в шарнирнике $\mathbf{p}^{\prime}$ в месте введения дополнительного ребра имеется два рычага, вполовину менее напряженных. Напряжения же всех остальных рычагов шарнирника $\mathbf{p}^{\prime}$ совпадают с напряжениями соответствующих рычагов $\mathbf{p .}$

Остается разобрать случай, когда граф $G_{\eta+1}$ получается из графа $G_{\eta}$ добавлением ребра, соединяюшего две несмежных в $G_{\eta}$ вершины. Этому и посвящена оставшаяся часть параграфа.

Чтобы завершить доказательство леммы 11, нам понадобятся следующие определения и ряд утверждений.

Совокупность $\left\{a_{1}, a_{2}, \ldots, a_{s}\right\}$ векторов пространства $\mathbb{R}^{n}$ назовем: положительно зависимой (ПЗ), если существует нетривиальная линейная комбинация $\sum_{i=1}^{s} \lambda_{i} a_{i}=0$, в которой все коэффищиенты $\lambda_{i} \geqslant 0 ;$ минимально положительно зависимой (МПЗ), если никакая ее собственная подсовокупность при этом не является положительно зависимой (такие системы векторов рассматривались, например, в [7]); и строго положительно зависимой (СПЗ), если существует равная нулю линейная комбинация, все коэффициенты которой положительны.

Очевидно, любая ПЗ система векторов содержит МПЗ совокупность, а любая МПЗ система векторов является СПЗ. Геометрически МПЗ совокупность векторов характеризуется следующим образом [7]: если взять начала всех ее векторов в точке $O \in \mathbb{R}^{n}$, то выпуклая оболочка их конщов будет представлять собой $(s-1)$-мерный симплекс, содержащий внутри себя точку $O$. Для МПЗ совокупности $\mathbf{A}=\left\{a_{1}, a_{2}, \ldots, a_{s}\right\}$, очевидно, все коэффищиенты линейной комбинации

$$
\sum_{i=1}^{s} \lambda_{i} \mathbf{a}_{i}=\mathbf{0}
$$

одного знака. Кроме того, это единственная, с точностью до умножения на число, нетривиальная комбинация векторов МПЗ системы $\mathbf{A}$, равная нулю. Из сказанного вытекает, что МПЗ систему векторов можно определить как такую, для которой положительность одного коэффициента в равенстве (а) влечет положительность всех остальных.

УТВЕРЖДЕНИЕ 6. Пусть $\mathbf{B}=\left\{\mathbf{b}_{1}, \ldots, \mathbf{b}_{p}\right\}-$ произвольная совожупность векторов, содержащаяся в линейной оболочке МПЗ системь $\mathbf{A} ;$ тогда $\mathbf{A} \cup \mathbf{B}$ - СПЗ совокупность векторов.

ДокАЗАТЕЛЬСТВО. Поскольку векторы $\mathbf{a}_{2}, \ldots, \mathbf{a}_{s}$ образуют базис линейной оболочки МПЗ системы векторов, сушествуют такие коэффициенты $\tau_{j}$, что

$$
\sum_{i=1}^{p} \mathbf{b}_{i}-\sum_{j=2}^{s} \tau_{j} \mathbf{a}_{j}=\mathbf{0} .
$$

Добавляя к этой линейной комбинации комбинацию (a), умноженную на достаточно большое число, можно получить комбинацию векторов совокупности $\mathbf{A} \cup \mathbf{B}$ со всеми положительными коэффициентами, равную нулю. 
УТВЕРЖДЕНИЕ 7. Совокупность векторов $\mathbf{A}=\left\{\mathbf{a}_{1}, \mathbf{a}_{2}, \ldots, \mathbf{a}_{s}\right\}$ евклидова пространства $\mathbb{R}^{n}$ является:

1) ПЗ в том и только том случае, когда для любого вектора $\mathbf{x} \in \mathbb{R}^{n}$ найдется такой вектор $\mathbf{a}_{k} \in \mathbf{A}$, что $\left(\mathbf{a}_{k}, \mathbf{x}\right) \geqslant 0$;

2) МПЗ тогда и только тогда, когда выполнено предыдущее условие и, кроме того, для любого $1 \leqslant j \leqslant s$ найдется такой вектор $\mathbf{x}$, что $\left(\mathbf{a}_{i}, \mathbf{x}\right)<0$ для всех $i \neq j$.

ДокАЗАТЕЛЬСтво. 1) НЕОБХОДИМОсть. Пусть совокупность векторов А ПЗ и комбинация (a) с $\lambda_{i} \geqslant 0$ нетривиальна. Тогда

$$
\sum_{i=1}^{s} \lambda_{i}\left(\mathbf{a}_{i}, \mathbf{x}\right)=\mathbf{0},
$$

и очевидно, что хотя бы одно из скалярных произведений в этой сумме обязано быть неотрицательным.

Достаточность. Допустим, что для совокупности А условие 1) не выполнено. Тогда существует такой вектор $\mathbf{x}$, что $\left(\mathbf{a}_{i}, \mathbf{x}\right)<0$ для любого $\mathbf{a}_{i} \in \mathbf{A}$, и совокупность векторов А не является ПЗ. Действительно, в этом случае невозможно выполнение равенства (б) со всеми неотрицательными коэффициентами, а следовательно, и равенства (а).

2) НЕОБХОдимость. Пусть совокупность А МПЗ и для определенности $j=s$. Тогда совокупность векторов $\left\{\mathbf{a}_{2}, \mathbf{a}_{3}, \ldots, \mathbf{a}_{s}\right\}$ линейно независима и ее можно дополнить до базиса $A$ в $\mathbb{R}^{n}$. Пусть $\left\{\mathbf{b}_{1}, \mathbf{b}_{2}, \ldots, \mathbf{b}_{n}\right\}=B$ - базис, сопряженный базису $A$. Тогда в качестве вектора $\mathbf{x}$, очевидно, можно взять вектор $-\sum_{i=1}^{n} \mathbf{b}_{i}$.

Достаточность. В силу первого условия совокупность $\mathbf{A}$ положительно зависима, а значит, существует нетривиальная комбинация $\sum_{i=1}^{s} \lambda_{i} \mathbf{a}_{i}=\mathbf{0}$, где $\lambda_{i} \geqslant 0$. Выберем какое-либо $j, 1 \leqslant j \leqslant s$, тогда вследствие этого равенства и второго условия существует такой вектор $\mathbf{x}$, что

$$
\lambda_{j}\left(\mathbf{a}_{j}, \mathbf{x}\right)=-\sum_{i \neq j} \lambda_{i}\left(\mathbf{a}_{i}, \mathbf{x}\right),
$$

и $\left(\mathbf{a}_{i}, \mathbf{x}\right)<0$ при $i \neq j$. Поскольку в силу второго условия среди векторов $\mathbf{a}_{i}$ нет нулевых, то хотя бы два из коэффициентов $\lambda_{i}, 1 \leqslant i \leqslant s$, отличны от нуля. Следовательно, правая часть последнего равенства отрицательна. А значит, с необходимостью $\lambda_{j}>0$. Так как выбор $j$ произволен, то $\lambda_{j}>0$ и, следовательно, совокупность А является МПЗ.

Пусть $p$ - общая точка гранищ выпуклых гладких тел $T_{1}, \ldots, T_{s}$ пространства $\mathbb{R}^{n}$, а $\mathbf{N}=\left\{\mathbf{n}_{1}, \ldots, \mathbf{n}_{s}\right\}$ - совокупность внешних нормалей этих тел в точке $p$; тогда справедливо следуюшее

УТВЕРЖДЕНИЕ 8. Совокупность нормалей $\mathbf{N}$ является:

1) ПЗ тогда и только тогда, когда $\operatorname{dim} \bigcap_{i=1}^{s} T_{i}<n$;

2) МПЗ тогда и только тогда, когда выполнено предыдущее условие и, кроме того, для любого $1 \leqslant j \leqslant s \quad \operatorname{dim} \bigcap_{i \neq j} T_{i}=n$. 
ДоКАЗАТЕЛЬСТво. Справедливость этого утверждения вытекает из предыдущего утверждения и следуюшего очевидного замечания. Если из точки $р$ границы гладкого выпуклого тела $T_{i}$ провести луч $L$ такого направления $\mathbf{x}$, что $\left(\mathbf{x}, \mathbf{n}_{i}\right)<0$, то все точки луча $L$, достаточно близкие к $p$, лежат внутри тела $T_{i}$. Если же направление $\mathbf{x}$ луча $L$ таково, что $\left(\mathbf{x}, \mathbf{n}_{i}\right) \leqslant 0$, то на луче $L$ нет точек, лежащих внутри $T_{i}$.

Рассмотрим в $\mathbb{R}^{d}$ псевдо-ЗШС $\mathscr{Z}$, имеющую $l$ свободных шарниров. Каждому шарнирнику р с псевдо-ЗШС $\mathscr{Z}$ в пространстве параметров $\mathbb{R}^{d l}=\mathbb{R}_{1}^{d} \oplus \mathbb{R}_{2}^{d} \oplus \cdots \oplus \mathbb{R}_{l}^{d}$ отвечает набор тел $\mathbf{U}_{i j}(\mathbf{p})=\mathbf{U}_{i j}$, задающихся неравенствами $\left(\mathbf{x}_{i}-\mathbf{x}_{j}\right)^{2} \leqslant d_{i j}$, где $\mathbf{x}_{i}$ - вектор подпространства $\mathbb{R}_{i}^{d}, d_{i j}=d_{i j}(\mathbf{p})-$ квадрат длины рычага $v_{i} v_{j}$ шарнирника $\mathbf{p}$. Если шарнирник $\mathbf{p}$ несократим, то каждое из тел $\mathbf{U}_{i j}$ представляет собой гладкий выпукльй цилиндр над $d$-мерным шаром радиуса $\sqrt{d_{i j}}$. Точка $\mathbf{p} \in$ $\mathbb{R}^{d l}$, очевидно, лежит в пересечении границ всех цилиндров $\mathbf{U}_{i j}, v_{i} v_{j} \in E(E-$ множество рычагов псевдо-ЗШС $\mathscr{Z})$.

УТВЕРЖДЕНИЕ 9. Шарнирник $\mathbf{p}$ является паутинным в том и только том случае, когда совокупность всех внешних нормалей $\mathbf{n}_{i j}(\mathbf{p}) \kappa$ иилиндрам $\mathbf{U}_{i j}$ в общей точке $\mathbf{p}$ их границ является СПЗ. Кроме того, в әтом случае пересечение $\Pi=\bigcap_{v_{i} v_{j} \in E} \mathbf{U}_{i j}$ состоит из одной лииь точки $\mathbf{p}$.

ДокАЗАТЕЛЬСтво. Строки координат векторов $\mathbf{n}_{i j}(\mathbf{p})$ являются строками матрицы Якоби $d \mathscr{F}(\mathbf{p})$ рычажного отображения $\mathscr{F}$, отвечающего псевдо-ЗШС $\mathscr{Z}$. Условие же

$$
\sum_{v_{i} v_{j} \in E} \omega_{i j} \mathbf{n}_{i j}(\mathbf{p})=\mathbf{0}
$$

равносильно совокупности условий (3) равновесия сил в свободных шарнирах. Отсюда следует справедливость первого из утверждений. Для доказательства второго заметим, что утверждения теоремы 4 не потеряют силы, если в ее условия заменить ЗШС на псевдо-ЗШС.

Допустим теперь противное второму из утверждений: $\Pi \ni \mathbf{p}^{\prime} \neq \mathbf{p}$. Тогда очевидно,

$$
d_{i j}\left(\mathbf{p}^{\prime}\right) \leqslant d_{i j}(\mathbf{p}), \quad v_{i} v_{j} \in E
$$

Если допустить, что все $d_{i j}\left(\mathbf{p}^{\prime}\right)=d_{i j}(\mathbf{p})$, то это будет противоречить однократности точки $\mathbf{d}=\mathscr{F}(\mathbf{p})$ при отображении $\mathscr{F}$, имеющей место вследствие расширенной теоремы 4. Допущение же справедливости какого-либо из неравенств $d_{i j}\left(\mathbf{p}^{\prime}\right)<d_{i j}(\mathbf{p})$ противоречит тому, что плоскость $\sum_{v_{i} v_{j} \in E} \omega_{i j} d_{i j}=\mathscr{C}$, где $\mathscr{C}$ - const (все $\omega_{i j}>0$ ), проходящая через точку $\mathbf{d}=\left\{d_{i j}\right\}$, опорна к множеству $\mathscr{C}=\mathscr{F}\left(\mathbb{R}^{d l}\right)$.

Наконец, мы можем завершить доказательство леммы 11. Будем считать, что псевдо-ЗШС $\mathscr{Z}_{\eta}$ и $\mathscr{Z}_{\eta+1}$ имеют по $l$ свободных шарниров. Тогда им обоим можно сопоставить одно и то же пространство параметров $\mathbb{R}^{d l}$. Перенумеруем цилиндры $\mathbf{U}_{i j}(\mathbf{p})$, отвечающие шарнирнику $\mathbf{p}$ с псевдо-ЗШС $\mathscr{Z}_{\eta}$, каким-либо об- 
разом: $\mathbf{U}_{1}(\mathbf{p}), \ldots, \mathbf{U}_{e}(\mathbf{p})$. Тогда шарнирнику $\mathbf{p}$ с теми же положениями шарниров, что и у предыдущего, но с псевдо-ЗШС $\mathscr{Z}_{\eta+1}$, соответствует набор цилиндров $\mathbf{U}_{1}(\mathbf{p}), \ldots, \mathbf{U}_{e}(\mathbf{p}), \mathbf{U}_{e+1}(\mathbf{p})$. Последний из этих цилиндров отвечает условию, налагаемому добавляемым к $\mathscr{Z}_{\eta}$ рычагом. По условию леммы 11 будем считать шарнирник $\mathbf{p}$ с псевдо-ЗШС $\mathscr{Z}_{\eta}$ паутинным. Тогда, вследствие утверждения 9, совокупность $\left\{\mathbf{n}_{1}(\mathbf{p}), \ldots, \mathbf{n}_{e}(\mathbf{p})\right\}$ внешних нормалей к этим цилиндрам является СПЗ. Но совокупность нормалей $\mathbf{N}=\left\{\mathbf{n}_{1}(\mathbf{p}), \ldots, \mathbf{n}_{e}(\mathbf{p}), \mathbf{n}_{e+1}(\mathbf{p})\right\}$ может оказаться (если последний вектор не лежит в линейной оболочке предыдущих) не СПЗ, и тогда точке $\mathbf{p} \in \mathbb{R}^{d l}$ не будет отвечать паутинный шарнирник с псевдо-ЗШС $\mathscr{Z}_{\eta+1}$.

Мы покажем, что найдется сколь угодно близкая к точке $\mathbf{p}$ в $\mathbb{R}^{d l}$ точка $\mathbf{p}^{\prime}$, которой отвечает паутинный шарнирник с псевдо-ЗШС $\mathscr{Z}_{\eta+1}$. Для этого понадобится следующее

УТВЕРЖДЕНИЕ 10. Пусть совокупность $\mathbf{N}_{s}=\left\{\mathbf{n}_{1}(\mathbf{p}), \ldots, \mathbf{n}_{s}(\mathbf{p})\right\}$ внешних нормалей к иилиндрам $\mathbf{U}_{1}(\mathbf{p}), \ldots, \mathbf{U}_{s}(\mathbf{p})$ является МПЗ, а нормаль $\mathbf{n}_{s+1}(\mathbf{p})$ не лежит в линейной оболочке совокупности $\mathbf{N}_{s}(u$, следовательно, совокупность $\left\{\mathbf{n}_{1}(\mathbf{p}), \ldots, \mathbf{n}_{s}(\mathbf{p}), \mathbf{n}_{s+1}(\mathbf{p})\right\}$ не является СПЗ и МПЗ). Обозначим $\mathbf{U}_{i}^{\prime}$ иилиндр в $\mathbb{R}^{d l}$ на $\varepsilon_{i}$, большего, чем $\mathbf{U}_{i}(\mathbf{p})$, радиуса и соосный с ним. Тогда для произвольного $\varepsilon>0$ найдется такой набор чисел $\varepsilon_{1}, \ldots, \varepsilon_{s+1},\left|\varepsilon_{i}\right|<\varepsilon$, $1 \leqslant i \leqslant s+1$, что $\bigcap_{i=1}^{s+1} \mathbf{U}_{i}^{\prime}$ состоит из одной лишь точки $\mathbf{p}^{\prime}$, а совокупность внешних нормалей $\left\{\mathbf{n}_{1}^{\prime}, \ldots, \mathbf{n}_{s+1}^{\prime}\right\} \kappa$ иилиндрам $\mathbf{U}_{i}^{\prime}$ в точке $\mathbf{p}^{\prime}$ является МПЗ.

ДокАЗАТЕЛЬСтво. Назовем касательным конусом $K$ выпуклого множества $M$ в точке его гранищы $\mathbf{x}$ замыкание объединения всех лучей, выходящих из х и пересекающих $M$ хотя бы еще в одной, кроме х, точке. Если граница полномерного множества $M$ гладкая, то касательный конус в каждой точке его гранищы есть замкнутоеполупространство. Очевидно, выпуклые множества $M_{1}$ и $M_{2}$, имеющие общую точку гранищ $\mathbf{x}$, пересекаются по внутренним точкам тогда и только тогда, когда их касательные конусы, проведенные в точке х, имеют общую внутреннюю точку.

Рассмотрим $(s-1)$-мерную плоскость $L^{s-1}$, проходящую через точку р и натянутую на нормали $\mathbf{n}_{1}(\mathbf{p}), \ldots, \mathbf{n}_{s}(\mathbf{p})$. Поскольку пересечения $\bigcap_{i \neq j}^{s} \mathbf{U}_{i}$ полномерны вследствие МПЗ совокупности векторов $\mathbf{N}_{s}$, их касательные конусы $K_{j}$ в точке $\mathbf{p}$ представляют собой полномерные цилиндры над $(s-1)$-гранными углами в плоскости $L^{s-1}$. Ясно, что пересечения конусов $K_{j}$ с касательным конусом цилиндра $\mathbf{U}_{s+1}$ в точке $\mathbf{p}$, являюшимся полупространством, полномерны. Отсюда следует, что и все пересечения $\mathbf{P}_{j}=\bigcap_{i \neq j}^{s+1} \mathbf{U}_{i}(1 \leqslant j \leqslant s, 1 \leqslant i \leqslant s+1)$ полномерны. Более того, $\varepsilon$ можно выбрать настолько малым, что при переходе от цилиндров $\mathbf{U}_{i}$ к цилиндрам $\mathbf{U}_{i}^{\prime}$ соответствуюшие пересечения $\mathbf{P}_{j}^{\prime}, \quad 1 \leqslant j \leqslant s$, останутся полномерными. Если взять $\varepsilon_{1}, \ldots, \varepsilon_{s}$ положительными, то пересечение $\mathbf{P}_{s+1}^{\prime}$ будет содержать внутри себя точку $\mathbf{p}$, и также будет полномерным. Остается показать, что $\varepsilon_{s+1}$ можно выбрать таким, чтобы пересечение $\mathbf{P}_{s+1}^{\prime} \cap \mathbf{U}_{s+1}^{\prime}$ состояло из одной лишь точки $\mathbf{p}^{\prime}$, и при этом было $\left|\varepsilon_{s+1}\right|<\varepsilon$. Последнее немедленно вытекает из следуюшего очевидного утверждения. Пусть замкнутые выпуклые множества 
$M_{1}, \ldots, M_{s}$ пересекаются в одной лишь точке, а $M_{i}^{\prime}$ - объединение всех замкнутых шаров радиуса $\varepsilon_{i}$ с центрами в точках множества $M_{i}$. Тогда если $\left|\varepsilon_{i}\right|<\delta$, то диаметр пересечения множеств $M_{1}^{\prime}, \ldots, M_{s}^{\prime}$ стремится к нулю при $\delta \rightarrow 0$. Утверждение 10 доказано.

Итак, выберем из ПЗ совокупности векторов $\mathbf{N}=\left\{\mathbf{n}_{1}(\mathbf{p}), \ldots, \mathbf{n}_{e}(\mathbf{p}), \mathbf{n}_{e+1}(\mathbf{p})\right\}$ МПЗ совокупность $\left\{\mathbf{n}_{i_{1}}(\mathbf{p}), \ldots, \mathbf{n}_{i_{s}}(\mathbf{p})\right\}$. Если линейная оболочка последней совокупности содержит совокупность $\mathbf{N}$, то система $\mathbf{N}$ является СПЗ, а шарнирник $\mathbf{p}$ с псевдо-ЗШС $\mathscr{Z}_{\eta+1}$ паутинным. В противном случае, пользуясь утверждением 10 , можно, последовательно нарашивая, скажем, $t$ раз, размерность МПЗ совокупности, перейти к такой точке $\mathbf{p}^{*}$ и таким цилиндрам $\mathbf{U}_{i}^{*}$, что линейная оболочка МПЗ совокупности $\left\{\mathbf{n}_{i_{1}}\left(\mathbf{p}^{*}\right), \ldots, \mathbf{n}_{i_{s}}\left(\mathbf{p}^{*}\right), \ldots, \mathbf{n}_{i_{s+1}}\left(\mathbf{p}^{*}\right)\right\}$ будет содержать в себе совокупность $\mathbf{N}^{*}=\left\{\mathbf{n}_{1}\left(\mathbf{p}^{*}\right), \ldots, \mathbf{n}_{e}\left(\mathbf{p}^{*}\right), \mathbf{n}_{e+1}\left(\mathbf{p}^{*}\right)\right\}$ нормалей всех цилиндров.

Вследствие утверждения 6 совокупность $\mathbf{N}^{*}$ СПЗ, а шарнирник $\mathbf{p}^{*}$ с псевдо-ЗШС $\mathscr{Z}_{\eta+1}$, в силу утверждения 9, паутинный. Поскольку точку $\mathbf{p}^{*}$ можно выбрать сколь угодно близкой к $\mathbf{p}$, то мы вправе считать шарнирник p* несократимым, если таковым был шарнирник p. Тем самым лемма 11, а вместе с ней и теорема 5 доказаны.

Из теоремы 5 вытекает

СЛЕДСТВИЕ 3. Приведенной ЗШС тогда и только тогда отвечает хотя бы один несократимый восстановимый по своему пространству напряжений шарнирник, когда число ее закрепленных шарниров больще 1 и ее дополненный граф $G^{\prime}$ не содержит разделяющих вершин.

В силу теоремы 5 достаточно установить, что каждый несократимый шарнирник с ЗШС, не удовлетворяющей условию следствия, невосстановим по своему пространству напряжений. Это сразу вытекает из следствия 2. Действительно, в качестве множества $V_{2}$ можно взять либо единственный закрепленный, либо один из разделяюших шарниров.

Заметим еще, что в случае, когда дополненный граф $G^{\prime}$ ЗШС $\mathfrak{Z}$ содержит разделяюшие вершины и распадается на $t$ неодносвязных компонент $G_{1}, G_{2}, \ldots, G_{t}$ (см. теорему $\left.1^{\prime}\right)$, то множество $\mathscr{C}=\mathbf{F}\left(\mathbb{R}^{d m}\right)$ представляет собой прямую сумму множеств $\mathscr{C}_{1}, \mathscr{C}_{2}, \ldots, \mathscr{C}_{t}$.

Опишем эти множества. Пусть $m_{i}$ - число свободных шарниров, $r_{i}-$ число рычагов в подграфе $G_{i}$, а $\mathbf{F}_{i}: \mathbb{R}^{d m_{i}} \rightarrow \mathbb{R}^{r_{i}}$ - рычажное отображение, отвечающее этому подграфу. Тогда $\mathbb{R}^{r}=\mathbb{R}^{r_{1}} \oplus \mathbb{R}^{r_{2}} \oplus \cdots \oplus \mathbb{R}^{r_{t}}$, а $\mathscr{C}_{i}=\mathbf{F}_{i}\left(\mathbb{R}^{d m_{i}}\right) \subset \mathbb{R}^{r_{i}}$. Причиной этого является возможность такого упорядочения множества $G_{1}, G_{2}, \ldots, G_{t}$ подграфов, что каждый следующий подграф пересекается с объединением предыдуших по одной лишь вершине. Это влечет то обстоятельство, что длины рычагов подшарнирника, отвечающего произвольному подграфу $G_{i}$, принимают значения никоим образом не зависяшие от длин рычагов подшарнирников, отвечаюших остальным подграфам. 


\section{Заключение}

Как отмечалось, паутинные шарнирники всегда восстановимы уже по одному внутреннему напряжению $\omega^{0}, \omega_{i j}^{0}>0$. В других простейших случаях это обстоятельство также имеет место: если шарнирник $\mathbf{p}$ восстановим по своему пространству $W(\mathbf{p})$ внутренних напряжений, то и в случае $\operatorname{dim} W(\mathbf{p})>1$ найдется такое напряжение $\omega^{0} \in W(\mathbf{p})$, что $\operatorname{det} \Omega\left(\omega^{0}\right) \neq 0$. Возникает естественный вопрос: всегда ли это так? Если шарнирник $\mathbf{p}$ восстановим по своему пространству напряжений $W(\mathbf{p})$, то всегда ли найдется такое напряжение $\omega^{0} \in W(\mathbf{p})$, что $\operatorname{det} \Omega\left(\omega^{0}\right) \neq 0$ ?

Для восстановимости каждого несократимого шарнирника с заданной ЗШС на прямой по его пространству напряжений необходимо и достаточно выполнения простых условий на его ЗШС, сформулированных в теореме 1. (Кроме всего прочего, эти условия гарантируют и полную напрягаемость несократимого шарнирника с такой ЗШС.) Пример $2 \S 3$ указывает, что на плоскости дело обстоит сложнее. Тем не менее, на примере $1 \S 3$ видно сушествование в плоскости ЗШС, для которых все несократимые полностью напрягаемые шарнирники восстановимы по своим пространствам напряжений. Задача состоит в том, чтобы попытаться описать все такие ЗШС на плоскости и в пространствах высшей размерности.

\section{Список литературы}

1. Ковалев М. Д. Геометрическая теория шарнирных устройств // Изв. РАН. Сер. матем. 1994. Т. 58. № 1. С. 45-70.

2. Connelly R. Rigidity and Energy // Invent. Math. 1982. V. 66. № 1. P. 11-33.

3. Уилсон Р. Введение в теорию графов. М.: Мир, 1977.

4. Crapo H., Whiteley W. Statics of Frameworks and Motions of Panel Structures, a Projective Geometric Introduction // Structural Topology. 1982. № 6. P. 43-82.

5. Постников М. М. Гладкие многообразия. М.: Наука, 1987.

6. Connelly $R$. The Rigidity of Certain Cabled Frameworks and the Second-Order Rigidity of Arbitrarily Triangulated Convex Surfaces // Advances in Math. 1980. V. 37. № 3. P. 272-299.

7. Болтянский В. Г., Солтан П. С. Комбинаторная геометрия различных классов выпуклых множеств. Кишинев: Штиинца, 1978. 